\title{
A Thermodynamically-Based Mesh Objective Work Potential Theory for Predicting Intralaminar Progressive Damage and Failure in Fiber-Reinforced Laminates
}

\author{
Evan J. Pineda * \\ NASA Glenn Research Center, Cleveland, OH, 44135, U.S.A. \\ Anthony M.Waas ${ }^{\dagger}$ \\ University of Michigan, Ann Arbor, MI, 48109, U.S.A.
}

\begin{abstract}
A thermodynamically-based work potential theory for modeling progressive damage and failure in fiber-reinforced laminates is presented. The current, multiple-internal state variable (ISV) formulation, enhanced Schapery theory (EST), utilizes separate ISVs for modeling the effects of damage and failure. Damage is considered to be the effect of any structural changes in a material that manifest as pre-peak non-linearity in the stress versus strain response. Conversely, failure is taken to be the effect of the evolution of any mechanisms that results in post-peak strain softening. It is assumed that matrix microdamage is the dominant damage mechanism in continuous fiber-reinforced polymer matrix laminates, and its evolution is controlled with a single ISV. Three additional ISVs are introduced to account for failure due to mode I transverse cracking, mode II transverse cracking, and mode I axial failure. Typically, failure evolution (i.e., post-peak strain softening) results in pathologically mesh dependent solutions within a finite element method (FEM) setting. Therefore, consistent character element lengths are introduced into the formulation of the evolution of the three failure ISVs. Using the stationarity of the total work potential with respect to each ISV, a set of thermodynamically consistent evolution equations for the ISVs is derived. The theory is implemented into commercial FEM software. Objectivity of total energy dissipated during the failure process, with regards to refinements in the FEM mesh, is demonstrated. The model is also verified against experimental results from two laminated, T800/3900-2 panels containing a central notch and different fiber-orientation stacking sequences. Global load versus displacement, global load versus local strain gage data, and macroscopic failure paths obtained from the models are compared to the experiments.
\end{abstract}

\section{Introduction}

Predictive capabilities of numerical tools for progressive damage and failure analysis (PDFA) of composite structures are predicated upon the robustness, accuracy, and objectivity of the tools. As sophisticated numerical tools become more feasible for widespread use in industry, significant weight and cost saving in the design of lightweight composite structures will be apparent. Virtual testing of materials, incorporating PDFA, can be used to evaluate the viability of materials or configurations prior to further scrutiny via physical testing. This grants analysts more flexibility during preliminary structural design stages and will ultimately manifest as more efficient and cost effective designs.

Continuum damage mechanics (CDM) has emerged as a viable option for predicting the non-linear behavior of composite structures. The first CDM theory was developed by Refs. 1,2. Subsequently, many publications on this subject were produced, including numerous books. ${ }^{3-7}$ Typically, a set of scalar damage

*Aerospace Research Engineer, Mechanics and Life Predictions Branch, 21000 Brookpark Rd., AIAA member.

${ }^{\dagger}$ Professor, Department of Aerospace Engineering, 1320 Beal Ave., AIAA fellow. 
variables, or internal state variables (ISVs), introduce anisotropic damage into the composite constituent behavior by penalizing the components of the material stiffness tensor, and non-linear functions are used to control the damage evolution. Various authors have used crack density, geometry, strain energy release rate, and other crack features to characterize the damage evolution. ${ }^{8-16}$ Others postulate damage evolution laws and characterize those laws using experiments. ${ }^{17-22}$ CDM models must also employ failure criteria to indicate damage initiation. More recently, increasingly sophisticated failure criteria have been developed to better represent the phenomenological behavior of a damaging composite lamina ${ }^{23-25}$ and used in conjunction with CDM.

CDM techniques offer computationally efficient and readily implementable means to capturing the effects of damage and failure in composite materials. Unfortunately, the majority of the criteria and evolution laws are formulated upon phenomenological observations of the directional dependence of damage evolution, rather than modeling the physics of the actual damage mechanisms. Separate damage variables are used to degrade different components of the stiffness tensor (directions) depending on whether the damage is said to accrue in the matrix or fiber constituents of the composite, but the variables do not explicitly distinguish between the separate damage mechanisms. Furthermore, many theories involve a multitude of parameters that are difficult to measure and must be calibrated to correlate with experimental data.

When implemented within the finite element method (FEM), many PDFA methodologies that utilize CDM breakdown when the material enters the post-peak strain softening regime locally within an element. Loss of positive definiteness of the tangent stiffness tensor leads to pathological mesh dependence. ${ }^{26,27}$ To overcome this deficiency, Ref. 28 developed the smeared crack, or crack band, model that introduces a characteristic element length into the formulation of the damage evolution. The original formulation assumed that the mode I crack band always aligns with the principle axes. ${ }^{29}$ Ref. 30 altered the formulation to accommodate a fixed crack band under mixed mode conditions. An encompassing overview of smeared crack band models is provided by Ref. 31 .

A thermodynamically-based, work potential theory, known as Schapery theory (ST), was developed for modeling matrix microdamage in fiber-reinforced laminates (FRLs). ${ }^{32-35}$ Refs. 36 and 37 extended the formulation to include the effects of transverse cracking by adding an additional internal state variable (ISV) and predicted the evolution of microdamage and transverse cracking in coupon laminates analytically. ${ }^{38}$ implemented this extended formulation in a numerical setting to simulate the failure of a buffer stripreinforced, center-notched panel (CNP). However, due to the cumbersome nature of the evolution equations, the microdamage and transverse cracking evolution equations were decoupled to arrive at a more efficient implementation. Since no characteristic length is introduced into the formulation, the theory produces mesh-dependent results in a computational setting.

The ST formulation is modified here, resulting in the enhanced Schapery theory (EST), to include the effects of macroscopic transverse and shear matrix cracking, as well as fiber breakage, using an approach that differs from Refs. 36-38. A deliberate distinction between damage and failure is made. Damage is defined as the effects of any structural changes resulting in a non-linear response that preserves the positive definiteness of the tangent stiffness tensor of the material. Conversely, failure is considered to be the consequence of structural changes that cause post-peak strain softening in the stress versus strain response of the material. Here, matrix microdamage is categorized as a damage mechanism, but macroscopic matrix cracking and fiber breakage are hypothesized to be failure mechanisms resulting from damage localization. The traditional ISV used in ST is maintained to model microdamage. Upon failure initiation, the element domain is no longer considered a continuum, and a smeared crack approach is used to model the embedded discontinuities. Three new ISVs, which incorporate the characteristic length of the finite element, dictate the evolution of the failure mechanisms. The EST formulation presented in Section II offers non-linear progressive damage coupled with mesh objective, post-peak strain softening.

Mesh objectivity is demonstrated in Section III. In Section IV, EST is verified against experimental results for two center-notched panels (CNPs). Global load versus deflection data, local strain gage data, as well as observed failure mechanisms obtained from experiments performed at the NASA Langley Research Center (LaRC) and exhibited in Refs. 39 and 40 are compared to numerical results.

\section{Enhanced Schapery Theory}

The previously developed $\mathrm{ST}^{34,37,38,41-43}$ is extended to accommodate mesh objective, post peak strain softening. Separate ISVs are used to govern the evolution of matrix microdamage, transverse (mode I) 
matrix failure, shear (mode II) matrix failure, and fiber breakage (mode I). The first and second laws of thermodynamics are enforced, establishing thermodynamically consistent evolution laws for progressive matrix microdamage, as well as post-peak failure. The following sections detail the formulation of this work potential theory.

\section{II.A. Thermodynamically-based Work Potential Framework}

As a material is loaded, a measure of the work potential facilitates modeling structural changes in the material, such as microcracking, which affect the elastic properties of the material. Energy that is not dissipated is recovered when the structure is unloaded, and the magnitude of energy recovered is contingent upon the degraded, elastic properties at the previously attained maximum strain state. It is assumed, upon subsequent reloading, that the material behaves linearly, exhibiting the elastic properties observed during unloading, until the material reaches the preceding maximum strain state. After this state is achieved, structural changes resume, affecting degradation of the instantaneous elastic moduli of the material. This process is shown in the uniaxial stress-strain curve displayed in figure 1. The shaded area above the unloading line represents total dissipated potential $W_{S}$, and the triangular area underneath is the total elastic strain energy density $W_{E}$. It is assumed that the material behaves as a secant material and there is no permanent deformation upon unloading. This a reasonable assumption for FRLs Ref. 36; however, plastic deformation can also be incorporated, if necessary. ${ }^{34}$ Extension to treat viscoelastic and viscoplastic response is outlined in Ref. 44.

Both $W_{E}$ and $W_{S}$ are functions of a set of ISVs, $S_{m},(m=1,2, M)$. These ISVs account for any inelastic structural changes in the material. Differentiating $W_{S}$ with respect to any ISV $S_{m}$, assuming limited pathdependence, ${ }^{34}$ yields the thermodynamic force, $f_{m}$, available for advancing structural changes associated with the $m^{\text {th }}$ ISV.

$$
f_{m}=\frac{\partial W_{s}}{\partial S_{m}}
$$

It is shown in Refs. 33 and 34 that the total work potential is stationary with respect to each ISV.

$$
\frac{\partial W_{T}}{\partial S_{m}}=0
$$

Additionally, Ref. 45 utilized the second law of thermodynamics to establish the inequality:

$$
f_{m} \dot{S}_{m} \geq 0
$$

which suggests that "healing" is not allowed for a material undergoing structural changes. Eqs. (1), (2), and (3) form the foundation of a thermodynamically-based work potential theory for modeling non-linear structural changes in a material exhibiting limited path-dependence.

\section{II.B. Multiple ISV Formulation of ST to Account for Multiple Damage and Failure Mecha- nisms}

Due to the generality of the evolution equations, Eqs. (2) and (3), the work potential theory can account for any number and type of structural changes that may occur in a material. This is especially useful for modeling progressive damage in composites because the heterogeneity of the composite, and multiaxiality of the local fields, enables multiple damage mechanisms to arise during a typical loading history. For instance in the matrix phase alone, microdamage accrues until its effects are superseded by the growth of larger transverse cracks. Microdamage is considered the advancement of microcracks, voids, fissures, shear bands, and other flaws that are present in the matrix of a composite ${ }^{36,37,42,46}$ The size of these flaws is typically on the order of that of the fiber or smaller. Transverse cracks nucleate from pre-existing flaws within the matrix but grow parallel to the fibers and span the thickness of the lamina..$^{9,10,14,17,47-49}$ Often, the growth of individual transverse cracks is extremely rapid; however, the effects of transverse cracking on the stiffness of a composite laminate can be progressive if multiple cracks form over an extended period of time and throughout an expansive volume. Eventually, transverse cracking is succeeded by more catastrophic damage mechanisms including interlaminar delamination, fiber breakage, pullout and bridging associated with macroscopic laminate fracture. ${ }^{15,50,51}$

The present EST formulation assumes that three major intralaminar mechanisms are responsible for all observed non-linearities in the stress-strain curve of a composite lamina: matrix microdamage, matrix 
macroscopic cracking, and axial fiber failure. Each of these mechanisms can be accommodated by partitioning the total dissipated energy density, $W_{S}$, into portions associated with each mechanism.

Matrix microdamage is the primary cause of observed non-linearity in the stress versus strain response of many polymer matrix composites (PMCs) (i.e. systems exhibiting negligible non-linear elasticity, plasticity or viscous effects) up to localization of microdamage into more severe failure mechanisms, such as transverse cracking, fiber breakage, kink band formation, or delamination. Microdamage can be considered the combination of matrix microcracking, micro-void growth, shear banding, and fiber-matrix debonding. figure 2 shows a typical uniaxial response of a material exhibiting microdamage evolution, where the recoverable energy potential is given by $W$ and the potential dissipated into evolving structural changes associated with microdamage is given by $S$.

Typically, matrix microdamage continues to grow until the onset of more catastrophic failure mechanisms initiate. It should be noted that this work explicitly distinguishes between damage and failure in the following manner:

Damage - Structural changes in a material that manifest as pre-peak non-linearity in the stress-strain response of the material through the degradation of the secant moduli.

Failure - Structural changes that result from damage localization in a material and manifest as post-peak strain softening in the stress-strain response of the material.

Here, three major failure mechanisms, which are distinct from the microdamage mode, are considered: transverse (mode I) matrix cracking, shear (mode II) matrix cracking, and axial (mode I) fiber fracture. These failure modes are consistent with the in-plane failure typically observed in PMC laminates. It is assumed that the evolution of these mechanisms yields an immediate reduction in the load-carrying capability of a local subvolume where the mechanism is active. Three ISVs are used to account for mode I matrix cracking, mode II matrix cracking, and mode I fiber failure, respectively: $S_{I}^{m}, S_{I I}^{m}$, and $S_{I}^{f}$. These ISVs are defined completely in Section II.C, and are taken to be the potentials required to advance structural changes associated with these failure mechanisms.

At any given state the total dissipated energy density $W_{S}$ can be calculated as a sum of energy dissipated through the aforementioned damage and failure mechanisms, given by the four ISVs.

$$
W_{S}=S+S_{I F}^{m}+S_{I I F}^{m}+S_{I}^{f}
$$

According to the first law of thermodynamics, the total work potential (ignoring thermal dissipation) is given by the sum of the elastic strain energy density and the potentials associated with each of the damage or failure mechanisms.

$$
W_{T}=W_{E}+S+S_{I}^{m}+S_{I I}^{m}+S_{I}^{f}
$$

where $W_{E}$ is the elastic strain energy density. Invoking the stationarity principle, Eq. (2),

$$
\begin{aligned}
& \frac{\partial W_{E}}{\partial S}=-1 \\
& \frac{\partial W_{E}}{\partial S_{I}^{m}}=-1 \\
& \frac{\partial W_{E}}{\partial S_{I I}^{m}}=-1 \\
& \frac{\partial W_{E}}{\partial S_{I}^{f}}=-1
\end{aligned}
$$


and the Second Law of Thermodynamics, Eq. (3), gives:

$$
\begin{aligned}
\dot{S} & \geq 0 \\
\dot{S}_{I F}^{m} & \geq 0 \\
\dot{S}_{I I F}^{m} & \geq 0 \\
\dot{S}_{F}^{f} & \geq 0
\end{aligned}
$$

Eqs. (6) and (7) constitute the evolution equations for damage and failure in a material associated with matrix microdamage, matrix cracking, and fiber breakage in tension.

It should be noted, that EST can also account for kink band formation under axial compression; $; 1,42,52$ although, the applied loading in the examples presented in Sections III and IV are tensile, and kink banding does not occur. As the lamina is loaded, the fibers in the composite rotate by some angle $\phi$, given by the deformation gradient in the model. To model the kink band mechanism, all calculations are then executed in the instantaneous fiber frame given by $\phi$; therefore, fibers rotation induces larger shear strains, $\gamma_{12}$. Increased shear strain yields more damage, leading to a reduction in the shear modulus. The increase in shear compliance allows for further progression of the shear strain. Under axial compression, this leads to a runaway instability, and a kink band will form.

\section{II.C. Failure Initiation}

Matrix microdamage requires no initiation criterion. For low strain levels, the microdamage ISV $S$ remains small and its effects on the composite moduli are not apparent. As $S$ evolves, with increased strains, its effects on the stress-strain response of the composite become more noticeable. However, it is postulated that the evolution of the failure mechanisms immediately yield a negative tangent stiffness; therefore, initiation criteria are required. Furthermore, criteria are required to mark failure initiation because the macroscopic cracks responsible for failure may result from localization of microdamage, or they may nucleate from preexisting flaws in the material not necessarily associated with microdamage.

EST is implemented in homogenized laminae; therefore, phenomenological criteria must be utilized that account for the composite microstructure. The Hashin-Rotem failure criterion incorporates separate equations for matrix failure and fiber failure initiation. ${ }^{53}$ The matrix failure criterion involves contributions from both the transverse $\left(\epsilon_{22}\right)$ and shear $\left(\gamma_{12}\right)$ strains.

$$
\begin{aligned}
& \left(\frac{\epsilon_{22}}{Y_{T}}\right)^{2}+\left(\frac{\gamma_{12}}{Z}\right)^{2}=1 \quad \epsilon_{22} \geq 0 \\
& \left(\frac{\epsilon_{22}}{Y_{C}}\right)^{2}+\left(\frac{\gamma_{12}}{Z}\right)^{2}=1 \quad \epsilon_{22}<0
\end{aligned}
$$

where $Y_{T}$ is the transverse lamina failure strain in tension, $Y_{C}$ is the transverse failure lamina strain in compression, and $Z$ is the shear failure strain. The fiber failure criterion only involves the axial strain $\epsilon_{11}$.

$$
\left(\frac{\epsilon_{11}}{X_{T}}\right)^{2}=1 \quad \epsilon_{11} \geq 0
$$

where $X_{T}$ is the maximium allowable axial strain of the lamina. A local, lamina coordinate frame is chosen such that, 1- is the axial direction of the fibers, 2- is the in-plane transverse direction, and 3- is the outof-plane direction. When Eq. (8) is satisfied the matrix failure ISVs $S_{I}^{m}$ and $S_{I I}^{m}$ are activated, and when Eq. (9) is satisfied fiber failure evolution $S_{I}^{f}$ is permitted; otherwise, $S$ remains the only active ISV. Upon satisfaction of either Eq. (8) or Eq. (9), it is assumed that the more severe failure mechanisms dominate, superseding the effects of matrix microdamage; therefore, $\dot{S}=0$, and additional microdamage is precluded. 


\section{II.D. Use of Traction-Separation Relationships to Define the Failure Potentials}

Refs. 36 and 37 used a single ISV to model the effects of transverse cracking on a composite lamina. Similar to microdamage, the transverse and shear moduli were related to transverse crack evolution through a set damage functions obtained from coupon experiments. Predictions of the non-linear response of numerous laminates were presented assuming a homogenous strain state in the laminates. Ref. 38 implemented the dual-ISV formulation of ST for predicting microdamage and transverse cracking within FEM to model the response of a center-notched laminate that was reinforced with buffer strips. The original formulation required the solution of two, coupled, bi-variate polynomials, which in an FEM framework became extremely computationally intensive. Thus, Ref. 38 decoupled the microdamage and transverse cracking evolution equations.

In the aforementioned publications, it was assumed that the transverse cracking affected the relationships between stress and strain. However, the existence of a macroscopic crack invalidates the assumption of a continuum. Here, it is presumed that failure arises from the evolution of cohesive cracks within the continuum, and the ISVs associated with failure (axial, transverse, and shear) influence the relationship between traction on the crack faces and the crack-tip opening displacement. The satisfaction of Eqs. (8) and/or (9) indicates the material behavior transitions from that of a damaging continuum to that of a cohesive crack, and the essential fields become traction and separation, rather than stress and strain (see figure 3).

Once a cohesive crack initiates in the continuum, opening of the crack yields a reduction in traction on the crack faces at the crack tip. If subsequently the crack is closed, it is assumed that traction at the crack tip will unload linearly towards the origin of the traction versus separation law (see figure 4). The strain energy release rate (SERR) $G_{M}^{j}$ is taken as the total energy dissipated per unit area of new surface that is created through crack advancement and can be calculated as the area under the traction-separation law (for a given traction and separation pair) minus the energy per area that can potentially be recovered by unloading.

$$
G_{M}^{j}=\int_{0}^{\delta_{M}^{j}} t_{M}^{j} d \delta_{M}^{j}-\frac{1}{2} t_{M}^{j} \delta_{M}^{j}
$$

where $j$ indicates the material (fiber $f$ or matrix $m$ ), $M$ represents the corresponding mode (mode I or mode II), $\delta_{M}^{j}$ is the crack tip opening displacement in mode $M$ and material $j$, and $t_{M}^{j}$ is the corresponding traction at the crack tip.

Theoretically, the shape of the traction-separation laws for mode I crack growth in the fiber, and mode I and II crack growth in the matrix can take any shape. ${ }^{54}$ investigated triangular, trapezoidal, beta distribution, and sinusoidal traction-separation laws in discrete cohesive zone method (DCZM) elements and determined that the shape only affected convergence of the FEM solver, but not the overall results. For simplicity, it is assumed here that all three types of cracks obey triangular traction-separation laws, presented in figure 4 . The total area under the traction-separation curves is controlled by the corresponding material fracture toughness in the appropriate mode, where $G_{I C}^{f}$ is the mode I fracture toughness of the fiber, $G_{I C}^{m}$ is the mode I fracture toughness of the matrix, and $G_{I I C}^{m}$ is the mode II fracture toughness of the matrix. The cohesive strengths of the materials $t_{I C}^{f}$ (mode I fiber strength), $t_{I C}^{m}$ (mode I matrix strength), and $t_{I I C}^{m}$ (mode II matrix strength) are given by the stresses in the continuum when Eqs. (8) and/or (9) are satisfied. Mode I, normal cracks are not allowed to grow under compression, but mode II, shear cracks can evolve under normal compression. Therefore, the mode I traction-separation laws for the fiber and matrix (figures $4 \mathrm{a}$ and $4 \mathrm{~b}$ ) do not accommodate negative crack tip displacements. However under negative mode II displacement (see figure 4c), the traction on the crack faces will increase linearly until the maximum, previously attained displacement magnitude is reached, after which, the crack faces will resume unloading according to the negative portion of the traction-separation law. The traction-separation laws exhibited in figure 4 do not require any initial, fictitious, pre-peak stiffness because the cracks are embedded within a continuum. This is an advantage over the use of DCZM elements which do require an initial stiffness because these interfacial elements do not actually represent physical material within the model and must attempt to simulate initially perfect bonding between adjacent material domains. ${ }^{55,56}$ If set incorrectly, these fictitious stiffnesses can cause numerical problems. ${ }^{57}$

Although no mode I crack can advance under compression, it is possible for post-peak softening to occur under compressive loading situations. For instance a kink band could form under global axial compression, or the matrix could fail in local shear due to internal friction (Mohr-Coulomb) in quasi-brittle materials 
under transverse compression. ${ }^{58}$ Since these failure mechanisms involve local shear at a the fiber/matrix scales which is typically below the operating lamina/laminate scale, it appears that these mechanisms evolve under mode I compression. In a model containing homogenized laminae, there is no subscale shear to drive these compressive failure modes. However, EST could be extended further to incorporate these mechanisms through phenomenological accessions. The methods, developed by ${ }^{42,52}$ and described in Section II.C, can be used to track the instantaneous fiber angle, and a critical fiber angle can be assigned to indicate the initiation of post-peak softening due to kink band formation. Similarly, a matrix compression criterion, such as the one developed by ${ }^{23,24}$ could be used to signal the initiation of Mohr-Coulomb compressive failure. The traction-separation laws for mode I fiber compression and mode I transverse matrix compression could be adjusted to include the post-peak softening effects of microbuckling and Mohr-Coulomb matrix failure. These postulated, compressive, mode I traction-separation laws could account for energy released through these subscale failure modes in a homogenous model at the lamina/laminate scale. However, the examples presented in this chapter are tension dominated, and extension of the theory to accommodate apparent mode I compressive failure is left for future work.

Using the traction-separation laws in figure 4, the SERR can be calculated with Eq. (10).

$$
\begin{aligned}
G_{I}^{f} & =\frac{1}{2} t_{I C}^{f} \delta_{I}^{f} \\
G_{I}^{m} & =\frac{1}{2} t_{I C}^{m} \delta_{I}^{m} \\
G_{I I}^{m} & =\frac{1}{2} t_{I I C}^{m} \delta_{I I}^{m}
\end{aligned}
$$

It is assumed that the energy released due to cracking is smeared over the entire element. ${ }^{28,29}$ Thus, the dissipation potentials in an element resulting from macroscopic cracking are related to the SERRs using the suitable element dimensions.

$$
\begin{gathered}
S_{I}^{f}=\frac{G_{I}^{f}}{l_{e}^{\left(\theta+90^{\circ}\right)}} \\
S_{I}^{m}=\frac{G_{I}^{m}}{l_{e}^{(\theta)}} \\
S_{I I}^{m}=\frac{G_{I I}^{m}}{l_{e}^{(\theta)}}
\end{gathered}
$$

If there is a single integration point in the element, $l_{e}^{\left(\theta+90^{\circ}\right)}$ is the length of a line running perpendicular to fiber direction in the element that intersects two edges of the element and the integration point, and $l_{e}^{(\theta)}$ is the length of a line that is parallel to the fiber direction in the element that intersects two edges of the element and the integration point. If there is more than one integration point in the element, the element can be partitioned into a number of subvolumes equal to the number of integration points, and the lengths

$l_{e}^{\left(\theta+90^{\circ}\right)}$ and $l_{e}^{(\theta)}$ are lengths of lines that intersect the corresponding integration point as well as two element edges or integration point subvolume boundaries. Incorporating a length scale into the ISVs results in mesh objective, post-peak, softening. This is elaborated upon further in Section IV.

\section{II.E. EST Evolution Equations for a Fiber-Reinforced Lamina}

To arrive at the evolution equations for the four ISVs, the elastic strain energy density must be defined for a material which may contain cohesive cracks. Therefore, the elastic strain energy $W_{E}$ is comprised of a contribution from the continuum $W$ and any possible cohesive cracks $W_{M}^{j}$. The plane stress, elastic strain energy density in the continuum is defined as

$$
W=\frac{1}{2}\left(E_{11} \epsilon_{11}^{2}+E_{22}(S) \epsilon_{22}^{2}+G_{12}(S) \gamma_{12}^{2}\right)+Q_{12} \epsilon_{11} \epsilon_{22}
$$

where stress in the laminae are related to strain assuming plane stress conditions.

$$
\begin{aligned}
& \sigma_{11}=Q_{11} \epsilon_{11}+Q_{12} \epsilon_{22} \\
& \sigma_{22}=Q_{12} \epsilon_{11}+Q_{22} \epsilon_{22} \\
& \tau_{12}=Q_{66} \gamma_{12}
\end{aligned}
$$


where $\gamma_{12}$ is the engineering shear strain and

$$
\begin{aligned}
Q_{11} & =\frac{E_{11}}{1-\nu_{12} \nu_{21}} \\
Q_{22} & =\frac{E_{22}}{1-\nu_{12} \nu_{21}} \\
Q_{12} & =\nu_{12} Q_{22} \\
Q_{66} & =G_{12} \\
\nu_{21} & =\frac{\nu_{12} E_{22}}{E_{11}}
\end{aligned}
$$

where $E_{11}$ is the axial elastic modulus, $E_{22}$ is the transverse elastic modulus, $\nu_{12}$ is the Poisson's ratio, $\nu_{21}$ is the transverse Poisson's ratio, and $G_{12}$ is the elastic shear modulus. After assuming that the quantity $\nu_{12} \nu_{21}<<1$, Eqs. (19) simplify,

$$
\begin{aligned}
& Q_{11}=E_{11} \\
& Q_{22}=E_{22} \\
& Q_{12}=\nu_{12} Q_{22} \\
& Q_{66}=G_{12}
\end{aligned}
$$

Note that only the transverse and shear moduli $\left(E_{22}\right.$ and $\left.G_{12}\right)$ are functions of $S$ since matrix microdamage only accrues in the matrix of the laminae. The Poisson's ratio is assumed to evolve such that the quantity $Q_{12}=E_{22} \nu_{12}$ remains constant; however, this restriction can be relaxed if deemed necessary. The degraded moduli are related to the virgin moduli $\left(E_{220}\right.$ and $\left.G_{120}\right)$ and the ISV through a set of microdamage functions $\left(e_{s}(S)\right.$ and $\left.g_{s}(S)\right)$ that are obtained from three uniaxial coupon tests. ${ }^{33,36,37}$

$$
\begin{aligned}
& E_{22}=E_{220} e_{s}(S) \\
& G_{12}=G_{120} g_{s}(S)
\end{aligned}
$$

Degrading $E_{22}$ and $G_{12}$ exclusively is consistent with the intralaminar damage typically observed in PMC laminates.

The elastic strain energy density of the cohesive cracks are defined as the recoverable energy per unit crack surface area smeared over the entire element.

$$
\begin{gathered}
W_{I}^{f}=\frac{t_{I}^{f} \delta_{I}^{f}}{2 l_{e}^{\left(\theta+90^{\circ}\right)}} \\
W_{I}^{m}=\frac{t_{I}^{m} \delta_{I}^{m}}{2 l_{e}^{(\theta)}} \\
W_{I I}^{m}=\frac{t_{I I}^{m} \delta_{I I}^{m}}{2 l_{e}^{(\theta)}}
\end{gathered}
$$

The tractions in Eqs. (23)-(25) can be related to the secant stiffness' in the traction-separation laws $k_{M}^{j}$.

$$
\begin{aligned}
t_{I}^{f} & =k_{I}^{f} \delta_{I}^{f} \\
t_{I}^{m} & =k_{I}^{m} \delta_{I}^{m} \\
t_{I I}^{m} & =k_{I I}^{m} \delta_{I I}^{m}
\end{aligned}
$$

Hence, the total elastic strain energy density in the continuum is given by

$$
\begin{aligned}
W_{E}= & \frac{1}{2}\left(E_{11} \epsilon_{11}^{2}+E_{22}(S) \epsilon_{22}^{2}+G_{12}(S) \gamma_{12}^{2}\right)+Q_{12} \epsilon_{11} \epsilon_{22} \\
& +\frac{k_{I}^{f}\left(S_{I}^{f}\right)}{2 l_{e}^{\left(\theta+90^{\circ}\right)}} \delta_{I}^{f^{2}}+\frac{k_{I}^{m}\left(S_{I}^{m}\right)}{2 l_{e}^{(\theta)}} \delta_{I}^{m 2}+\frac{k_{I I}^{m}\left(S_{I I}^{m}\right)}{2 l_{e}^{(\theta)}} \delta_{I I}^{m 2}
\end{aligned}
$$

Substituting Eq. (29) into Eqs. (6) gives the ISV evolution equations.

$$
\frac{1}{2}\left(\epsilon_{22}^{2} E_{220} \frac{d e_{s}}{d S_{r}}+\gamma_{12}^{2} G_{120} \frac{d g_{s}}{d S_{r}}\right)=-3 S_{r}^{2}
$$

$$
8 \text { of } 40
$$




$$
\begin{gathered}
\frac{1}{2 l_{e}^{\left(\theta+90^{\circ}\right)}} \frac{d k_{I}^{f}}{d S_{I}^{f}} \delta_{I}^{f^{2}}=-1 \\
\frac{1}{2 l_{e}^{(\theta)}} \frac{d k_{I}^{m}}{d S_{I}^{m}} \delta_{I}^{m 2}=-1 \\
\frac{1}{2 l_{e}^{(\theta)}} \frac{d k_{I I}^{m}}{d S_{I I}^{m}} \delta_{I I}^{m 2}=-1
\end{gathered}
$$

The use of a reduced ISV $S_{r}=S^{\frac{1}{3}}$ has been employed in Eq. (30). Ref. 36 has shown that the use of this reduced ISV yields polynomial forms of the microdamage functions in Eqs. (21) and (22). Using the chain rule and the fact that

$$
\begin{gathered}
\frac{d S_{I}^{f}}{d \delta_{I}^{f}}=\frac{t_{I C}^{f}}{2 l_{e}^{\left(\theta+90^{\circ}\right)}} \\
\frac{d S_{I}^{m}}{d \delta_{I}^{m}}=\frac{t_{I C}^{m}}{2 l_{e}^{(\theta)}} \\
\frac{d S_{I I}^{m}}{d \delta_{I I}^{m}}=\frac{t_{I I C}^{m}}{2 l_{e}^{(\theta)}}
\end{gathered}
$$

by Eqs. (11)-(16), the cohesive secant stiffnesses are determined.

$$
\begin{aligned}
k_{I}^{f} & =-\int \frac{t_{I C}^{f}}{\delta_{I}^{f^{2}}} d \delta_{I}^{f} \\
k_{I}^{m} & =-\int \frac{t_{I C}^{m}}{\delta_{I}^{m}{ }^{2}} d \delta_{I}^{m} \\
k_{I I}^{m} & =-\int \frac{t_{I I C}^{m}}{\delta_{I I}^{m^{2}}} d \delta_{I I}^{m}
\end{aligned}
$$

Evaluating the integrals in Eqs. (37)-(39), while enforcing $k_{M}^{j}=0$ when $\delta_{M}^{j}=\frac{2 G_{M C}^{j}}{t_{M C}^{j}}$ results in expressions for $k_{M}^{j}$ in terms of $\delta_{M}^{j}$.

$$
\begin{gathered}
k_{I}^{f}=t_{I C}^{f}\left(\frac{1}{\delta_{I}^{f}}-\frac{t_{I C}^{f}}{2 G_{I C}^{f}}\right) \\
k_{I}^{m}=t_{I C}^{m}\left(\frac{1}{\delta_{I}^{m}}-\frac{t_{I C}^{m}}{2 G_{I C}^{m}}\right) \\
k_{I I}^{m}=t_{I I C}^{m}\left(\frac{1}{\delta_{I I}^{m}}-\frac{t_{I I C}^{m}}{2 G_{I I C}^{m}}\right)
\end{gathered}
$$

The thermodynamically consistent stiffnesses derived in Eqs. (40)-(42) can also be derived directly from the traction-separation laws using geometry.

Finally, it is assumed that following failure initiation the strains are related to the crack tip opening displacements by

$$
\begin{gathered}
l_{e}^{\left(\theta+90^{\circ}\right)} \epsilon_{11}=l_{e}^{\left(\theta+90^{\circ}\right)} \epsilon_{11}^{C}+\delta_{I}^{f} \\
l_{e}^{(\theta)} \epsilon_{22}=l_{e}^{(\theta)} \epsilon_{22}^{C}+\delta_{I}^{m} \\
l_{e}^{(\theta)} \gamma_{12}=l_{e}^{(\theta)} \gamma_{12}^{C}+2 \delta_{I I}^{m}
\end{gathered}
$$

where $\epsilon_{11}^{C}, \epsilon_{22}^{C}$, and $\gamma_{12}^{C}$ are the strains when Eqs. (8) and/or (9) are satisfied. Eqs. (43)-(45) imply that the strain in the continuum remains at the values obtained when failure initiates, and that any incremental change in the global strain after failure initiation is used wholly to advance the crack tip opening displacement. To account for changes in the continuum strain after failure initiates, it can be assumed that the stress state in the cracked body is homogenous and the tractions on the crack tip faces are equal to the stresses in the continuum. Then, the strains in Eq. (29) can be formulated in terms of the cohesive secant stiffnesses and the crack tip opening displacement. However, it is assumed that the evolution of strain in the continuum 
is negligible once cohesive cracks form. Eqs. (43)-(45) can be utilized in Eqs. (40)-(42) to obtain $k_{M}^{j}$ as functions of the global strain at an integration point.

$$
\begin{gathered}
k_{I}^{f}=t_{I C}^{f}\left[\frac{1}{l_{e}^{\left(\theta+90^{\circ}\right)}\left(\epsilon_{11}-\epsilon_{11}^{C}\right)}-\frac{t_{I C}^{f}}{2 G_{I C}^{f}}\right] \\
k_{I}^{m}=t_{I C}^{m}\left[\frac{1}{l_{e}^{(\theta)}\left(\epsilon_{22}-\epsilon_{22}^{C}\right)}-\frac{t_{I C}^{m}}{2 G_{I C}^{m}}\right] \\
k_{I I}^{m}=t_{I I C}^{m}\left[\frac{2}{l_{e}^{(\theta)}\left(\gamma_{12}-\gamma_{12}^{C}\right)}-\frac{t_{I I C}^{m}}{2 G_{I I C}^{m}}\right]
\end{gathered}
$$

Once failure initiates, the effects of failure supersede the effects of microdamage and evolution of $S$ ceases. The cohesive stiffness in a cracked element is calculated using Eqs. (46)-(48) for a given strain state; then, Eqs. (26)-(28) and (43)-(45) are used to calculate the tractions on the crack tip faces and the crack tip opening displacement. It is assumed that the stress state in the integration point subvolume of the element is homogenous, and the tractions on the crack tip faces are equal to the stresses in the element. Lastly, the axial, transverse, and shear moduli of the element can be calculated: ${ }^{29}$

$$
\begin{gathered}
E_{11}=\left\{\frac{1}{E_{110}}-\frac{\epsilon_{11}-\epsilon_{11}^{C}}{t_{I C}^{f}\left[1+\frac{l_{e}^{\left(\theta+90^{\circ}\right)} t_{I C}^{f}}{2 G_{I C}^{f}}\left(\epsilon_{11}-\epsilon_{11}^{C}\right)\right]}\right\}^{-1} \\
E_{22}=\left\{\frac{1}{E_{22}^{*}}-\frac{\epsilon_{22}-\epsilon_{22}^{C}}{t_{I C}^{m}\left[1+\frac{l_{e}^{(\theta)} t_{I C}^{m}}{2 G_{I C}^{m}}\left(\epsilon_{22}-\epsilon_{22}^{C}\right)\right]}\right\}^{-1} \\
G_{12}=\left\{\frac{1}{G_{12}^{*}}-\frac{\gamma_{12}-\gamma_{12}^{C}}{2 t_{I I C}^{m}\left[1+\frac{l_{e}^{(\theta)} t_{I I C}^{m}}{4 G_{I I C}^{m}}\left(\gamma_{12}-\gamma_{12}^{C}\right)\right]}\right\}^{-1}
\end{gathered}
$$

where $E_{22}^{*}$ and $G_{12}^{*}$ are the degraded transverse and shear moduli, due to microdamage, when Eq. (8) is satisfied.

For visualization purposes in the FEM simulations, degradation parameters are defined which relate the current, degraded stiffnesses to their original values upon failure initiation.

$$
\begin{aligned}
& D_{I}^{f}=1-\frac{E_{11}}{E_{11}^{*}} \\
& D_{I}^{m}=1-\frac{E_{22}}{E_{22}^{*}} \\
& D_{I I}^{m}=1-\frac{G_{12}}{G_{12}^{*}}
\end{aligned}
$$

The degradation parameter can have a minimum value of zero, which indicates that no degradation has occurred, or a maximum value of one, signaling that the corresponding modulus has been completely diminished.

The negative tangent stiffness of the stress-strain curve necessary for post-peak strain softening to occur imposes a restriction the maximum allowable element size, as shown by. ${ }^{29}$

$$
l_{e}^{\left(\theta+90^{\circ}\right)}<\frac{2 G_{I C}^{f} E_{11}}{t_{I C}^{f}{ }^{2}}
$$




$$
l_{e}^{(\theta)}<\min \left\{\frac{2 G_{I C}^{m} E_{22}^{*}}{t_{I C}^{m}{ }^{2}}, \frac{2 G_{I I C}^{m} G_{12}^{*}}{t_{I I C}^{m}}\right\}
$$

The analyst must be careful to ensure the dimensions of any failing elements are smaller than the conditions given in Eqs. (55) and (56).

In summary, Eqs. (8) and (9) mark the transition from evolving microdamage to failure to macroscopic cracking. Prior to failure initiation, Eq. (30) is used to calculate the microdamage reduced ISV $S_{r}$, and the failure ISVs $S_{I}^{f}, S_{I}^{m}$, and $S_{I I}^{m}$ remain zero. Eqs. (21) and (22) are used to calculate the degraded transverse and shear moduli. Subsequent to matrix failure initiation, microdamage growth is precluded, and $S_{r}$ remains at $S_{r}^{*}$, the value of $S_{r}$ when Eq. (8) was satisfied. The degeneration of the transverse and shear moduli, resulting from matrix transverse and shear cracking, is calculated using Eqs. (50) and (51). Finally if Eq. (9) is satisfied, the axial modulus is calculated using Eqs. (49) as fiber breakage evolves in the element. Once the material moduli have been calculated using the appropriate evolution equations, the stresses can be updated accordingly using Eqs. (20).

\section{Mesh Objectivity}

The theory outlined in Section II eliminates the mesh dependency that arises from ill-posedness when the elements enter the post-peak softening regime by introducing a characteristic length into the formulation. The total SERR dissipated during the evolution of the discontinuity is equal to the prescribed fracture toughness and is independent of the element size. This approach, commonly referred to as the smeared crack approach, or crack band model, has been used to alleviate mesh dependency in FEM since it was first developed by ${ }^{28}$ for post-peak strain softening in concrete.

To exhibit the mesh objectivity of EST, a simple example is presented in this section. One quarter of a $200 \mathrm{~mm} \times 100 \mathrm{~mm}$ panel containing a central hole is modeled with finite elements using the Abaqus, version 6.10-1 finite element software. ${ }^{59}$ The panel contains a hole with a radius of $r_{h}=5 \mathrm{~mm}$ in the center. The left edge of the panel is constrained in the $x$-direction to simulate symmetry. Similarly, the bottom edge is constrained in the $y$-direction. A uniform displacement is applied to all the nodes on the top edge of the panel in $y$-direction. Details on the panel geometry and boundary conditions are displayed in figure 5 . The panel is composed of a generic, $\left[90^{\circ}\right]$, composite lamina with the fiber angle measured with respect to the $y$-axis; thus, the applied displacement is perpendicular to the fiber direction in the panel. EST is used to model damage and failure in the panel.

Four different meshes are used to evaluate the effect of mesh size on the response of the panel. All four meshes consist of two-dimensional (2-D), plane stress, quadrilateral, S4R shell elements. The elements are linear, reduced integration elements and contain four nodes and one integration point each. The density of the four meshes, shown in figure 6, increases within a region near the central hole. Average element sizes equal $0.5 r_{h}, 0.2 r_{h}, 0.1 r_{h}$, and $0.04 r_{h}$ are used in the four different meshes. Coarser elements are used away from the hole to improve computation time. The four meshes are subjected to the same boundary conditions and loading, and are composed of the same material properties. The same elastic, damage and failure parameters are also used in all four simulations.

The resultant, applied tensile stress (given by two times the sum of the reaction forces at the nodes on the top edge divided by the cross-sectional area) normalized by the transverse, mode I, critical strain times the transverse Young's modulus $\bar{\sigma}$ is plotted versus the applied displacement normalized by the radius of the hole $\bar{\Delta}$ for the four different meshes in figure 7 . It can be seen that the mesh density has a minimal effect on the load-deflection results. The small discrepancy in the results between the four meshes can be attributed to the increased accuracy in the fields as the mesh is refined. Moreover, the total energy dissipated is preserved from mesh to mesh.

Figure 8 displays contours of the normalized, reduced microdamage ISV $S_{r}$ immediately before failure initiation in the four different meshes. $S_{r}$ is normalized by the maximum $S_{r}$ obtained in all four simulations which is $27 \%$ of the maximum allowable $S_{r}$ required to bring the moduli to zero. The four meshes exhibit similar microdamage contours, but as the mesh is refined, the microdamage is contained to the vicinity of the hole. Additionally, the global stress at which failure initiates reduces slightly as the mesh is refined; this supports the previous statement that the discrepancies in the stress-displacement responses were a facet of increasing field accuracy with mesh refinement.

The transverse matrix failure degradation parameter $D_{I}^{m}$ is plotted for all four meshes in figure 9 . figures 9a-9d show the failure pattern at the ultimate, global load. The coarsest mesh shows that a crack band 
has grown at the intersection of the hole and the bottom symmetric boundary and is propagating towards the free edge, while moving away from the bottom boundary when the specimen ultimate load is achieved. In the finer meshes, the crack band path is different. In figures $9 \mathrm{~b}-9 \mathrm{c}$ the crack band initiates at the hole slightly above the bottom boundary and propagates towards the free edge while approaching the bottom boundary. Since crack band initiation is governed by the quadratic H-R failure criterion (Eq. (8)), both the transverse and shear strains are contributing to the initiation leading to crack band initiation at the hole boundary away from the symmetric, horizontal edge where the transverse strain is not the maximum. Any failure criterion, in theory, could be used to govern crack band initiation, and if a maximum strain criterion was used, the crack band would always initiate at the hole boundary at the symmetric edge of the model, which is the location of the maximum transverse strain. The finest mesh, figure $9 \mathrm{~d}$, exhibits multiple crack bands near the hole, but only one of the crack bands grows significantly. While in all simulations the first crack band initiates at the hole, figures $9 \mathrm{c}$ and $9 \mathrm{~d}$ show some crack bands beginning to initiate at the free edge and propagate inwards by the time the ultimate load is attained. Figures $9 \mathrm{e}-9 \mathrm{~h}$ display the transverse crack band pattern once the specimen has lost all load-carrying capability. The solution for the simulation with the finest mesh $0.04 r_{h}$ diverged before all the load carrying capability was lost; so, figure $9 \mathrm{~h}$ presents the crack band path at the final converged state, which is still far below the ultimate load state. In figures 9e-9h the same crack band patterns that developed in figures 9a-9d are evident, except those crack bands have saturated to maximum degradation: $D_{I}^{m}=1$. Additionally, the crack bands advancing from the free edge observed in figures $9 \mathrm{c}$ and $9 \mathrm{~d}$ have progressed further. However, this is well after the specimens reached their ultimate load; therefore, it is assumed that those crack bands did not influence the load carrying capability of the panels. The discrepancy in crack band path observed for the different meshes indicate that the crack band path is dictated by the accuracy of the fields surrounding the leading boundary of the crack band path.

\section{Example - Center Notched Panels Subjected to Uniaxial Tension}

\section{IV.A. Experimental Details}

Two center-notched panel (CNP) configurations were tested at the NASA Langley Research Center (LaRC). ${ }^{39,40}$ The geometrical details of the panel and testing boundary conditions are presented in figure 10. The panels were 3 " wide and 11.5 " long. Two 3" x 2.75 " tabs were placed on both ends of the specimens, leaving a gage section of 3" x 6" which is displayed in figure 10. A central notch was machined in each panel that was 0.75 " wide and had a notch tip radius of $0.09375 "$. The end tabs were clamped and a vertical, tensile displacement (in the $y$-direction) was applied to the top tab using a servo-hydraulic testing machine. The bottom tab was fixed preventing any $y$-displacement of the bottom boundary of the gage section. The gripped tabs also prevented any displacement in the $x$-direction at the top and bottom boundaries of the gage section.

The panels were comprised of laminated T800/3900-2 carbon fiber/toughened epoxy composites. Three different lay-up configurations were tested; however one of the configurations exhibited significant delamination. Since the focus of this work is modeling in-plane damage and failure mechanisms, this configuration is not considered here. The two remaining configurations are presented in table 1 . The first lay-up, Laminate-1, consists of $120^{\circ}$ plies, and the second, Laminate-2, is a symmetric, multi-angle lay-up with $40 \%\left|45^{\circ}\right|, 40 \%$ $0^{\circ}$, and $20 \% 90^{\circ}$ layers.

Several strain gages where affixed to the test panel, labeled Sg-1 through $\mathrm{Sg}-4$ in figure 10. Sg-1 was placed in the center of the panel, 1.5" above the notch. Sg-2 was placed 1.5" above the notch tip. Sg-3 was attached in front of the notch, 0.5" from the free edge, and Sg-4 placed at the notch tip. Global load versus displacement data, and local strain gage data was reported in Refs.,${ }^{39,40}$ along with a post-test C-Scan of Laminate-1 and photograph of Laminate-1 and photograph of Laminate-2.

\section{IV.B. Finite Element Model Details}

The linear elastic properties of T800/3900-2 used in the FEM models are presented in table 2, and were taken from Ref. 39. The shear microdamage function $g_{s}$ utilized in Eq. (22) was obtained from $\left[45^{\circ} /-45^{\circ}\right]_{3 S}$ angle-ply T800/3900-2 coupon tests. The transverse, tensile and compressive microdamage functions were inferred by scaling the coefficients of the microdamage curves presented by ${ }^{36}$ for AS4/3502 by the ratio of the virgin transverse modulus of T800/3900-2 to that of AS4/3502, as the stress-strain curves of the coupon 
laminates necessary to characterize $e_{s}$ were not available. The polynomial forms of $e_{s}$ and $g_{s}$ are

$$
\begin{aligned}
& e_{s}\left(S_{r}\right)=e_{s 0}+e_{s 1} S_{r}+e_{s 2} S_{r}^{2}+e_{s 3} S_{r}^{3}+e_{s 4} S_{r}^{4}+e_{s 5} S_{r}^{5} \\
& g_{s}\left(S_{r}\right)=g_{s 0}+g_{s 1} S_{r}+g_{s 2} S_{r}^{2}+g_{s 3} S_{r}^{3}+g_{s 4} S_{r}^{4}+g_{s 5} S_{r}^{5}
\end{aligned}
$$

The coefficients of the microdamage curves are presented in table 3 , and the curves are plotted in figure 12 .

To increase computational efficiency, the first derivatives of the higher order microdamage polynomials are approximated using linear spline interpolants. Thus, the microdamage evolution equation, Eq. (30), is always second order in $S_{r}$ yielding a very efficient analytical solution. Since the value of $S_{r}$ from the previous increment is used to estimate which spline regime should be used to solve for $S_{r}$ in the current increment, the solution is checked to ensure that it falls within the applicable range of the spline that was used. If it falls outside of the range of $S_{r}$ that are valid for the splines, the solution is calculated again using splines that accord to the solution of the previous iteration. This procedure continues until the solution of Eq. (30) falls within the relevant range of $S_{r}$ for the splines used in Eq. (30). A maximum number of iterations can be set, after which Eq. (30) is solved using the full polynomial forms of the damage functions, given in Eqs. (57) and (58), by finding the eigenvalues of the companion matrix of the polynomial coefficients of the evolution equation.

The axial mode I, transverse mode I, and shear mode II critical cohesive strains, and fracture toughness' are given in table 4 . The matrix mode I and mode II cohesive critical strains $\left(Y_{T}, Y_{C}\right.$, and $\left.Z\right)$ and the fracture toughnesses $\left(G_{I C}^{m}\right.$ and $\left.G_{I I C}^{m}\right)$ were calibrated using data from Laminate-1. These values were adjusted until the global load versus local strain at strain gage Sg-1 (see figure 10) obtained from the model provided the best match to the experimental data. Both the simulation results and experimental data are presented in figure 14a. Laminate-1 did not exhibit any axial failure; so, the fiber mode I parameters $\left(X_{T}\right.$ and $\left.G_{I C}^{f}\right)$ were calibrated such that the ultimate load from the simulation of Laminate- 2 corresponded with the ultimate load reported by ${ }^{39}$ for Laminate-2. Subsequent work will outline a procedure for measuring these values experimentally.

Displacement was applied to both laminates using the *DYNAMIC keyword in Abaqus with the parameter APPLICATION = QUASI-STATIC. This implicit dynamic solver is recommended for quasi-static problems exhibiting a high-degree of nonlinearity. This procedure uses numerical damping to stabilize the problem. The numerical damping does not significantly affect the simulation results because the velocities in these simulations are low. For Laminate- 1 a total displacement of 0.0236 ", and for Laminate-2 a displacement of 0.0472 ", is applied over 1000 seconds. The panels were assigned a representative density of $0.057 \mathrm{lb} / \mathrm{in}^{3}$. This implicit, dynamic technique has advantages over traditional static, implicit solvers which have difficulty converging when the material exhibits post-peak softening, ${ }^{60,61}$ and is not limited by a minimum stable time step required with explicit solvers. ${ }^{62}$

\section{IV.C. Results - Laminate-1}

Global load $P$ versus displacement $\Delta$ of a 4 " section of Laminate-1 is compared to results from the EST simulation in figure 13. Very good agreement between the model and the experimental results is achieved. The response of the specimen appears to be linear until near 8,000 lbf., where the specimen begins deforming non-linearly. The EST simulation captures the initiation and progression of the global nonlinearity accurately. This panel was not loaded until catastrophic failure; hence, the data presented in figure 13 represents load versus displacement data prior to the ultimate load of the specimen.

Local strain gage data (global load $P$ versus local $y$-direction strain $\epsilon_{y y}$ ) from Laminate-1 is plotted with the results from the EST FEM model in figure 14; please refer to figure 10 for locations of strain gages. Strain relaxation is observed in the gage farthest away from the notch: Sg-1, shown in figure 14a. The mode I and mode II matrix failure parameters in EST were calibrated such that the model demonstrates the same transition into strain relaxation at this location and at a similar global applied load. This load, taken as the splitting load, is 8,250 lbf. in experiment and 8,210 lbf. in the model (summarized in table $5)$. The transition to strain relaxation is more abrupt in the experiment as evidenced by the sharp knee in the load-strain curve, whereas, the transition in the model is more gradual. Prior to strain relaxation at this point, the experiment displayed slight stiffening not observed in the model. Additionally, the model response is much smoother than that of the experiment in the strain relaxation regime. Even though the global loading is quasi-static, local events, such as cracking, may be dynamic; therefore, the discrepancy in the strain relaxation portion of the load-strain curves could be a result of dynamic matrix crack growth and 
arrest in the test specimen. Local crack dynamics were not taken into account in the model. Additionally, the jaggedness of the experimental data may be a facet of the stochastics related to the local microstructure of the composite that are not included in the model. The data from the experiment and simulation for Sg-2, which is located 1.5" directly above the notch, are presented in figure 14b. The model predicts less strain at Sg-2, for a given load, than the experiment, but the non-linear trends are very similar. This gage lies directly in front of the splitting crack path, shown in figure 10, and it is not realistic to expect perfect agreement in areas experiencing high levels of damage and failure because of idealizations used to model the evolution of cracks in the simulations. Figure 14c displays data for $\mathrm{Sg}-3$, located in front of the notch near the free edge. Very good agreement between the experimental and simulation results are exhibited. The model accurately captures the non-linear evolution of strain, away from the highly damaged regions, as a function of applied load. Finally, results for Sg-4 (located directly at the notch tip) are given in figure 14d and includes the experiment and simulation display of axial strain relaxation. As with $\mathrm{Sg}-2, \mathrm{Sg}-4$ shows less strain for a given applied load. However, the load at which the strain at Sg-4 relaxes in both the experiment and model correlate well, in accordance with the splitting load. Again, the relaxation response of the experiment is discontinuous, but the model exhibits continuous behavior.

A C-Scan of the failed Laminate-1 specimen is displayed in figure 15. Four splitting cracks can be observed propagating outward from the notch tip, parallel to the loading direction, towards the gripped edges. Contour plots of the normalized microdamage obtained from the simulation are presented in figure 16 at the splitting load 8,200 lbf. and at 16,400 lbf. In these plots, $S_{r}$ is normalized by the maximum achievable value, $S_{r}^{\max }=7.57 \mathrm{psi}^{\frac{1}{3}}$, obtained from figure 12 . In Laminate-1, $S_{r}$ reached a maximum value equal to $0.171 S_{r}^{\max }$. At the splitting load, the regions of maximum damage are localized to small regions, along the same crack path observed in figure 15, embedded in a more widespread domain and exhibiting less severe microdamage. A similar microdamage contour is observed at $P=16,400 \mathrm{lbf}$., except the localized damage region has nearly proceeded to the fixed boundaries of the panel.

Figure 17 shows the shear failure degradation factor $D_{I I}^{m}$ at the splitting load and 16,400 lbf. The shear failure localizes into crack bands that are a single element wide and progress equivalently to the cracks observed in the experiment. At the splitting load (figure 17a), the crack bands have progressed less than half of the way between the notch and the panel boundary on either side of the notch. In figure $17 \mathrm{~b}$, the crack bands have nearly reached the fixed grip boundaries. Additionally, figure 17b displays some irregularity in the crack path. In these regions, the mesh is not uniform because it needed to accommodate larger elements used to represent the strain gages (see figure 11). No axial failure was observed in this simulation. The shear crack path was in a state of transverse compressions, and according to the traction-separation relations used

in figure $4 \mathrm{~b}$, transverse failure does not progress under compressive conditions. Thus, contours of $D_{I}^{f}$ and $D_{I}^{m}$ are not shown.

\section{IV.D. Results - Laminate-2}

Numerical results for applied load versus displacement of a 4" section of Laminate-2 are presented in figure 18. The experimental ultimate load $15,300 \mathrm{lbf}$. correlates well (axial failure parameters were calibrated to obtain an ultimate load that most closely matched the experimental data) with the ultimate load obtained from the model, also 15,300 lbf., and is summarized with the splitting load from the Laminate- 1 analysis in table 5. The global response up to failure is nearly linear and failure occurs suddenly and catastrophically.

Figure 19 compares the applied load versus strain gage results from the model to the data from the experiment. Sg-1 and Sg-2 exhibited similar behavior; the strain increases until the ultimate load is obtained, after which the strain relaxes abruptly. The experimental data and numerical results both display this behavior. The model exhibits slightly more strain, for a given load, prior to ultimate failure. At Sg-3, the model predicts strain localization after the ultimate load is achieved. The gage data shows a slight reduction in strain as the load drops; however, the gage was placed directly in the crack path and may have been damaged when the panel failed. The model results and experimental data for $\mathrm{Sg}-4$ exhibit similar trends, but the strain gage shows a large degree of nonlinearity at the notch tip. ${ }^{39}$ attributed this observed nonlinearity to local interlaminar stresses near the notch free edge which caused some local delaminations. Since the focus of this work was modeling in-plane damage mechanisms, these effects are not captured; however, the model could be easily extended to incorporate delamination by placing DCZM elements between continuum shell layers. ${ }^{40}$

In the experiment, the gages measure the strain over a continuous area associated with the size of the gage, but in the model, the strain is taken at the integration point of an element; thus, these measures should 
not be expected to correspond exactly. In areas where there are large gradients present, such as near a notch tip (Sg-4) or near cracks (Laminate-1, Sg-2 or Laminate-2, Sg-3), it becomes even more difficult to relate the strain gage data to numerical strains from a discretized continuum. This may contribute to some of the discrepancies between the local gage data and the model results in figures 14 and 19.

A photograph taken of the failed, Laminate-2 specimen is presented in figure 20 . The photograph shows that two macroscopic cracks initially propagate from the notch tip towards the free edges, perpendicular to the applied load, in a self-similar fashion. Eventually, the cracks turn and proceed towards the free edge at an angle. ${ }^{39}$ claim, supported by visual image correlation displacement data, that there was some eccentricity in the specimen alignment, which resulted in deviation from self-similar crack growth.

Normalized microdamage contours just prior to the ultimate load are presented for the outermost $45^{\circ}$, $0^{\circ},-45^{\circ}$, and $90^{\circ}$ plies in figure 21. Similar microdamage patterns are evident in the $45^{\circ}$ and $-45^{\circ}$ layers. Microdamage propagates outward, toward the free edge, from the notch tip in petal-like patterns. The microdamage in these layers is highly distributed throughout the plies. The $0^{\circ}$ ply displays a more contained microdamage pattern along the lines of the microdamage contours associated with axial splitting as shown in figure 16. A moderate level of microdamage is also displayed in the $90^{\circ}$ layer, but a low degree of microdamage is distributed throughout most of the layer.

Figure 22 shows the axial failure degradation parameter $D_{I}^{f}$ at the ultimate load for the four unique layers. A small amount of axial failure in the $45^{\circ}, 0^{\circ}$, and $-45^{\circ}$ layers can be observed at the notch tips. It appears that more failure occurs at one notch tip than the other. This can be attributed to numerical imperfections resulting from dissimilar meshes at the opposite notch tips, that is the mesh is not symmetric about the $y$-axis. No axial failure is observed in the $90^{\circ}$ layer. Contours of the transverse, mode I, failure degradation parameter $D_{I}^{m}$ at the ultimate load are plotted in figure 23. The failure patterns are similar in the $45^{\circ}$ and $-45^{\circ}$ plies in figures 23a and 23c and are comparable to the microdamage contours in figures 21a and 21c, except the failure is restricted to regions on either side of the notch. Furthermore, small, highly degraded domains can be observed propagating from the notch tip at an angle corresponding to the fiber direction in the ply. The $90^{\circ}$ layer exhibits some moderate degradation in a localized region around the notch tips, and the $0^{\circ}$ layer does not exhibit much $D_{I}^{m}$. Contours of the shear, mode II, failure degradation parameter $D_{I I}^{m}$ are presented at the ultimate load in figure 24 . Very similar failure paths can be seen in the $45^{\circ}$ and $-45^{\circ}$ layers and the patterns are nearly symmetric across both centerlines of the panel. This is expected because as figure $4 \mathrm{c}$ indicates, the sign of the local shear strain does not affect the failure degradation. $D_{I I}^{m}$ in the $0^{\circ}$ and $90^{\circ}$ is limited to very small regions surrounding the notch tips.

Contours representing the microdamage in the four unique layers are presented in figure 25 after the panel has completely failed and lost all of its load carrying capability. Although further matrix microdamage evolution is prohibited in elements that have failed (transverse/shear or axial), in the other elements that have not failed, matrix microdamage evolution continues. Nearly the entire $45^{\circ}$ and $-45^{\circ}$ layers reach a microdamage level of $0.18 S_{r}^{\max }$. The $0^{\circ}$ and $90^{\circ}$ plies exhibit similar microdamage patterns; however, low levels of microdamage are more widespread in the $90^{\circ}$ ply. figure 26 shows the fiber failure path once the specimen has completely failed. All of the layers, except the $90^{\circ}$ layer, show self similar cracks propagating from the notch tips towards the free edges of the panel. The angled crack path shown in figure 20 was not reproduced because the eccentric loading (suspected in the test) was not introduced into the simulation; therefore, the crack growth remained self-similar. A high degree of transverse matrix failure can be seen in the axial crack path in the $45^{\circ},-45^{\circ}$, and $90^{\circ}$ plies in figure 27 . In the $0^{\circ}$ layer, some transverse failure is observed surrounding the fiber failure, as well as away from the axial failure path, which resulted from a stress wave reflecting off the free edges when the axial crack band reaches the boundary. Finally, $D_{I I}^{m}$ is presented after the specimen has failed in figure 28. Similar failure to figures $24 \mathrm{a}$ and $24 \mathrm{c}$ in the $45^{\circ}$ and $-45^{\circ}$ is exhibited, but a highly degraded region has localized in the axial crack path. figures $28 \mathrm{~b}$ and $28 \mathrm{~d}$ show fairly extensive regions containing a high degree of shear matrix failure surrounding the axial failure path.

\section{Conclusions}

A thermodynamically-based, work potential theory for damage and failure in composite materials, enhanced Schapery theory (EST), was developed. A marked distinction between damage and failure was introduced. Damage was considered to be the evolution of mechanisms that cause structural changes in the material such that the non-linear tangent stiffness tensor remains positive definite. Failure was taken to be the effect of structural changes in the material that result in loss of positive definiteness of the tangent 
stiffness matrix and post-peak strain softening. Separate internal state variables (ISVs) were used to account for damage and three in-plane failure mechanisms.

In EST, matrix microdamage, which includes matrix microcracking, shear banding, and microvoid growth, is responsible for all damage in a composite lamina and was accounted for with a single ISV, along the lines of the original Schapery theory (ST) formulation. The relationship between the transverse and shear moduli of the lamina were related to the ISV through a pair of experimentally-obtainable microdamage functions.

Three major, in-plane failure mechanisms applicable to continuous fiber-reinforced, laminated, polymer matrix composites were identified: mode I matrix cracks, mode II matrix cracks, and fiber breakage. A failure initiation criterion was used to mark the transition from a damaging continuum to a damaged continuum with an embedded discontinuity. After failure initiation, microdamage evolution ceases and separate ISVs are introduced to incorporate the effects of the three major failure mechanisms. Evolution of the failure ISVs is based upon traction-separation laws (which are a functions of the appropriate fracture toughnesses) and a characteristic element length. Typically, the existence of a non-positive definite stiffness tensor would result in pathologically mesh dependent solutions; however, in EST, mesh objectivity is ensured by incorporating a characteristic length scale into the failure evolution.

In Section III of this paper, mesh objectivity is demonstrated. A unidirectional composite plate with a central hole obeying EST is loaded in transverse tension and the response is calculated using four different, increasingly refined, meshes. The global stress versus strain response remained unaffected by the change in mesh, save for the effects from increased accuracy of local fields in the vicinity of the hole with denser meshing.

Two center-notched panels, with different lay-ups, composed of T800/3900-2 were tested under tensile loading at NASA LaRC. Global load versus displacement and global load versus local strain gage strain data were compared to results obtained from FEM models utilizing EST in Section IV. Quantitatively, very good correlation was achieved for both laminates. Furthermore, damage and failure paths predicted by the models matched well with the experimental results. 


\begin{tabular}{|l|c|c|}
\hline ID & Stacking Sequence & Thickness (in.) \\
\hline \hline Laminate-1 & {$\left[0^{\circ}\right]_{12}$} & 0.078 \\
\hline Laminate-2 & {$\left[45^{\circ} / 0^{\circ} /-45^{\circ} / 0^{\circ} / 90^{\circ}\right]_{S}$} & 0.065 \\
\hline
\end{tabular}

Table 1: T800/3900-2 lay-up configurations used in CNP tests at NASA LaRC.

\begin{tabular}{|l|c|}
\hline Property & T800/3900-2 \\
\hline \hline$E_{11}(\mathrm{Msi})$ & 23.2 \\
\hline$E_{22}(\mathrm{Msi})$ & 1.3 \\
\hline$G_{12}(\mathrm{Msi})$ & 0.9 \\
\hline$\nu_{12}$ & 0.28 \\
\hline
\end{tabular}

Table 2: Linear elastic properties for T800/3900-2 used in FEM models.

\begin{tabular}{|c|c|l|c|c|}
\hline$e_{s}\left(S_{r}\right)$ Coefficients & \multicolumn{1}{|c|}{ Tension } & Compression & $g_{s}\left(S_{r}\right)$ Coefficients & \\
\hline \hline$e_{s 0}$ & 1.0000 & 1.0000 & $g_{s 0}$ & 1.0000 \\
\hline$e_{s 1}$ & $-6.0373 \mathrm{E}-2$ & $8.4887 \mathrm{E}-4$ & $g_{s 1}$ & $-3.0567 \mathrm{E}-2$ \\
\hline$e_{s 2}$ & $2.5937 \mathrm{E}-2$ & $2.8002 \mathrm{E}-2$ & $g_{s 2}$ & $-1.2135 \mathrm{E}-1$ \\
\hline$e_{s 3}$ & $-1.5789 \mathrm{E}-2$ & $-6.2122 \mathrm{E}-3$ & $g_{s 3}$ & $3.7438 \mathrm{E}-2$ \\
\hline$e_{s 4}$ & $2.2571 \mathrm{E}-3$ & N/A & $g_{s 4}$ & $-4.5405 \mathrm{E}-4$ \\
\hline$e_{s 5}$ & $-1.0440 \mathrm{E}-4$ & N/A & $g_{s 5}$ & $1.9532 \mathrm{E}-4$ \\
\hline
\end{tabular}

Table 3: Microdamage function coefficients for T800/3900-2 used in FEM models. 


\begin{tabular}{|c|l|}
\hline Property & T800/3900-2 \\
\hline \hline$X_{T}$ & 0.021 \\
\hline$Y_{T}$ & 0.0092 \\
\hline$Y_{C}$ & 0.0115 \\
\hline$Z$ & 0.0075 \\
\hline$G_{I C}^{f}$ & $1026 \frac{\mathrm{lbf} . \text {-in. }}{\text { in. }^{2}}$ \\
\hline$G_{I C}^{m}$ & $2.39 \frac{\text { lbf.-in. }}{\text { in. }^{2}}$ \\
\hline$G_{I I C}^{m}$ & $6.78 \frac{\text { lbf.-in. }}{\text { in. }{ }^{2}}$ \\
\hline
\end{tabular}

Table 4: Failure parameters for T800/3900-2.

\begin{tabular}{||c|c|c|c|}
\hline & Type & Experimental & Numerical \\
\hline \hline Laminate-1 & Splitting & $8,250 \mathrm{lbf}$. & $8,210 \mathrm{lbf}$. \\
\hline Laminate-2 & Ultimate & $15,300 \mathrm{lbf}$. & $15,300 \mathrm{lbf}$. \\
\hline
\end{tabular}

Table 5: Critical experimental and simulation loads for Laminate-1 and Laminate-2.

18 of 40 


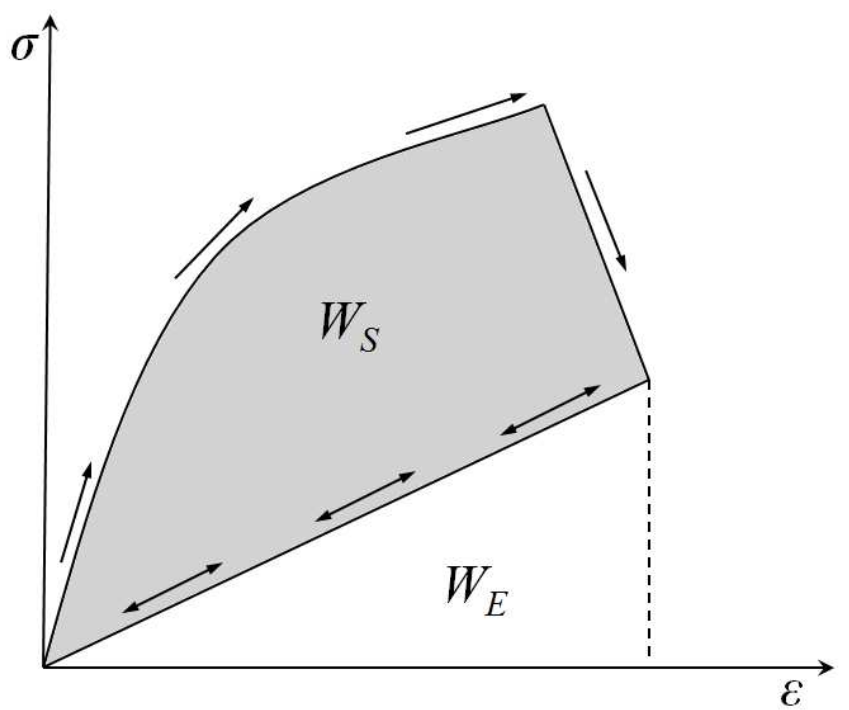

Figure 1: Typical stress-strain curve, containing pre-peak nonlinearity and post-peak strain softening, showing the total elastic $\left(W_{E}\right)$ and total dissipated $\left(W_{S}\right)$ potentials.

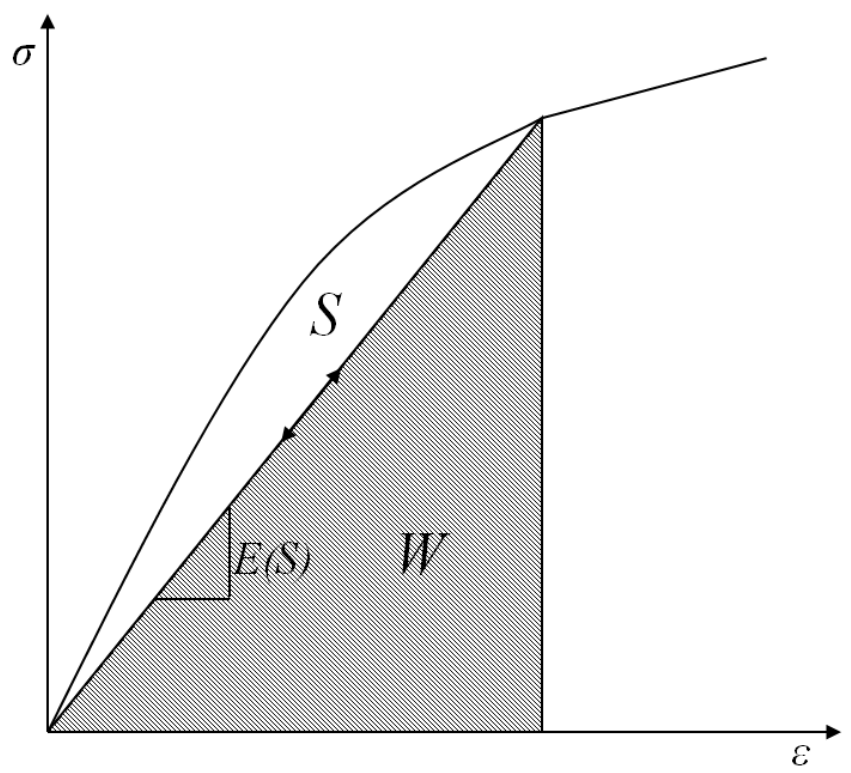

Figure 2: Typical stress-strain curve with a positive-definite tangent stiffness exhibiting microdamage, showing the elastic $(W)$ and irrecoverable $(S)$ portions. 


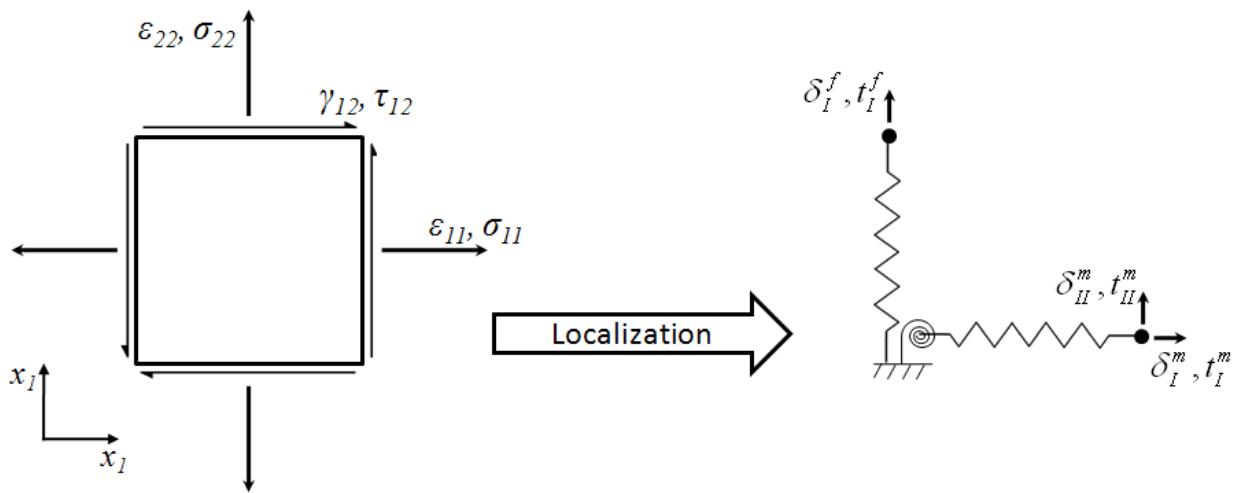

Figure 3: Schematic showing the transition form a continuum to a cohesive zone due to the initiation of macroscopic cracks. The essential, constitutive variables switch from stress and strain to traction and separation.

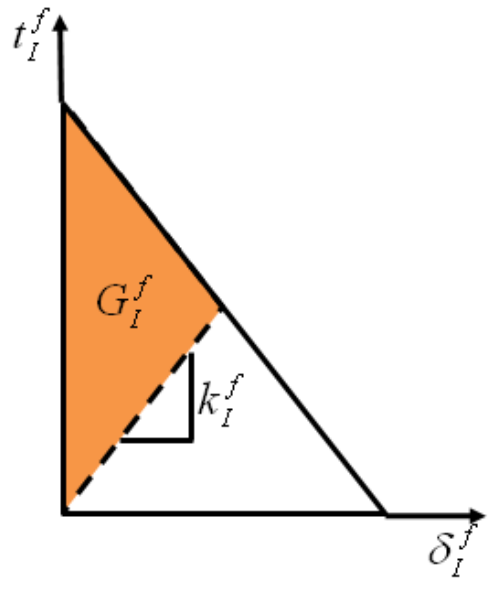

(a) Mode I fiber fracture.

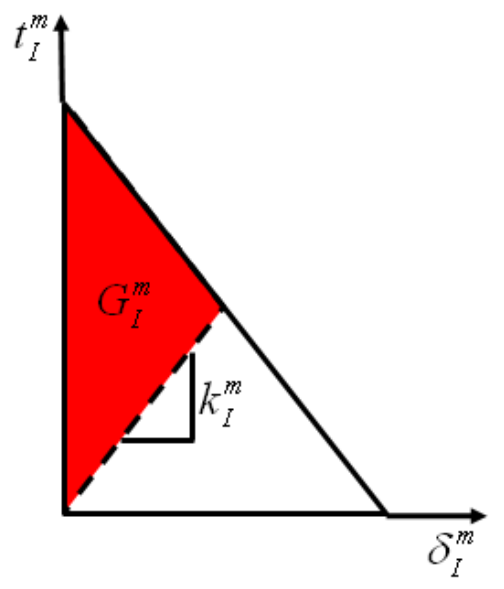

(b) Mode I matrix fracture.

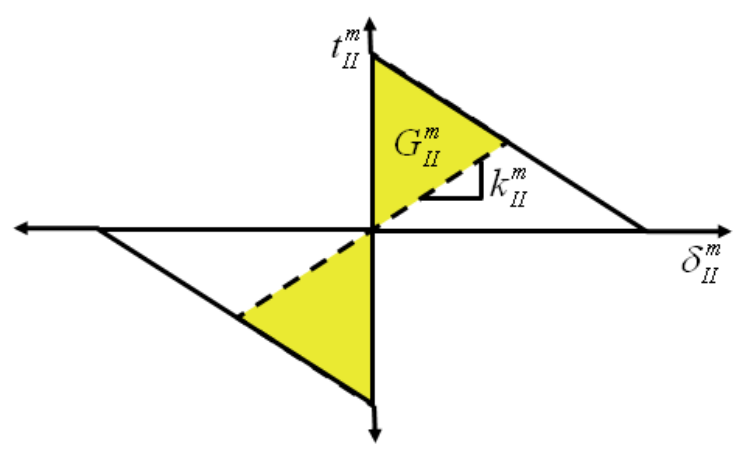

(c) Mode II matrix fracture.

Figure 4: Triangular traction versus separation which dictates the behavior of cohesive cracks embedded in the continuum. The total area under the traction-separation law represents the material fracture toughness $G_{m C}^{j}$. The area above the unloading line for a given traction-separation state is the strain energy release rate $G_{m}^{j}$. 


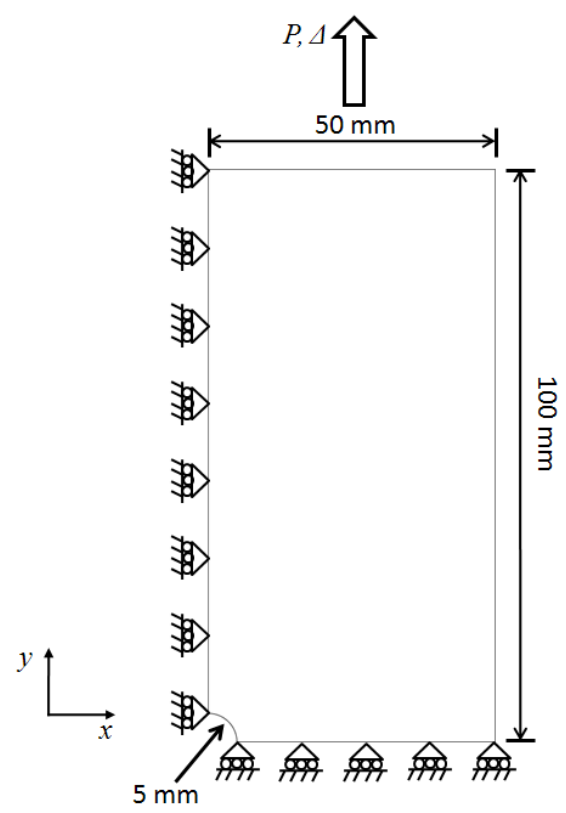

Figure 5: Example problem used to demonstrate mesh objectivity of EST. One quarter of a $200 \mathrm{~mm} \times 100$ $\mathrm{mm}$ containing a central hole with a radius of $5 \mathrm{~mm}$ is loaded in tension with symmetric boundary conditions on the bottom and left boundaries.

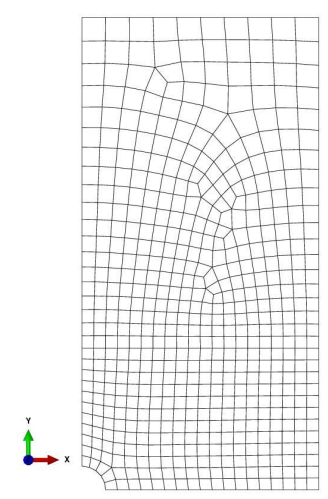

(a) $0.5 r_{h}$.

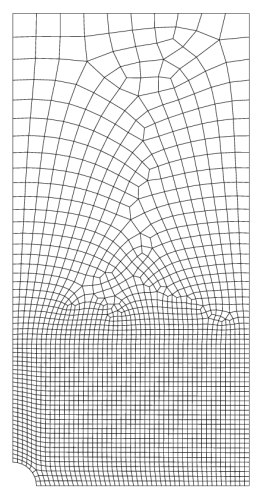

(b) $0.2 r_{h}$.

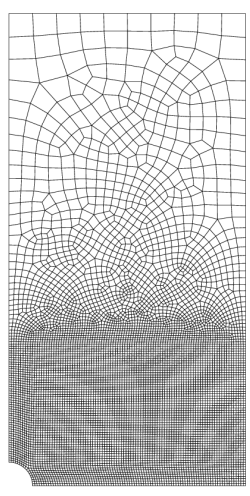

(c) $0.1 r_{h}$.

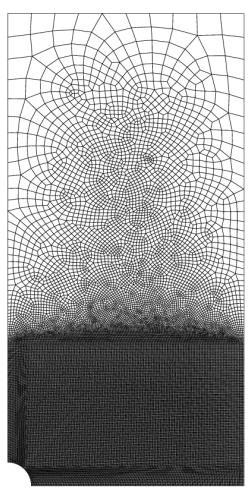

(d) $0.04 r_{h}$.

Figure 6: Four mesh densities used to demonstrate the mesh objectivity of EST. 


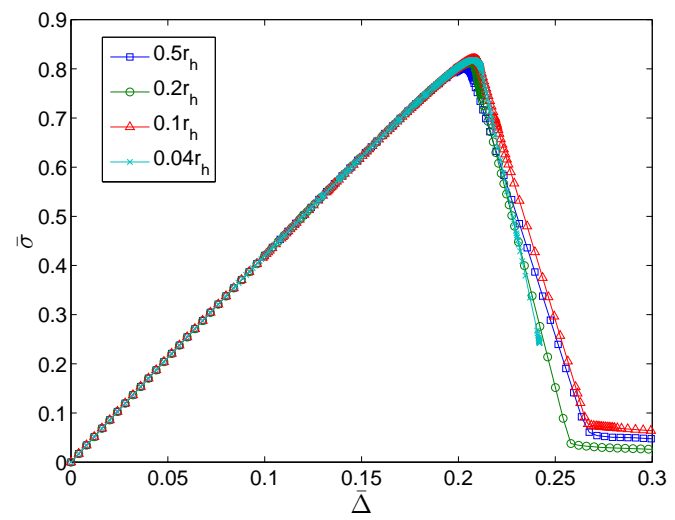

Figure 7: Reaction stress normalized by critical axial strain times axial Young's modulus verse applied displacement normalized by hole radius for four different mesh densities.

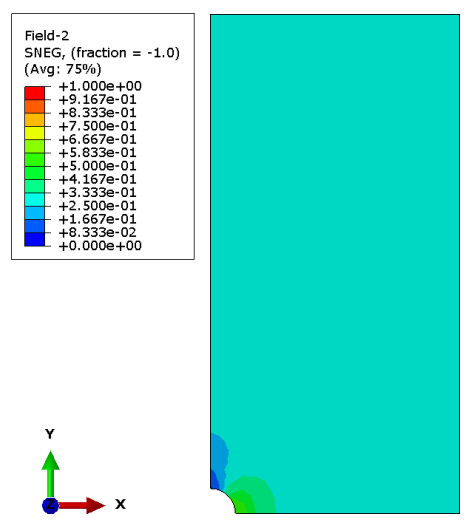

(a) $0.5 r_{h}, \bar{\sigma}=0.50$.

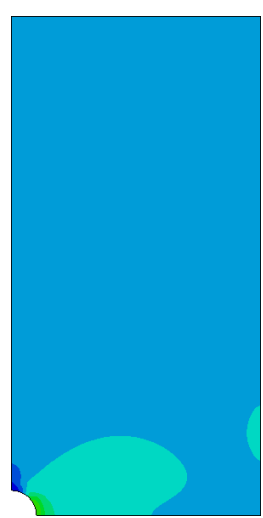

(b) $0.2 r_{h}, \quad \bar{\sigma}=$ 0.42 .

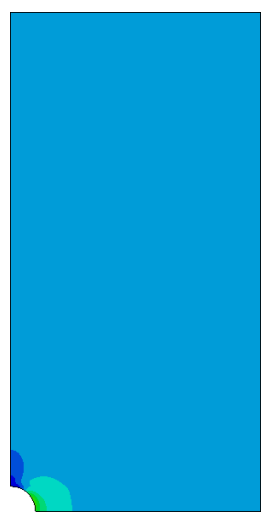

(c) $0.1 r_{h}, \bar{\sigma}=0.37$.

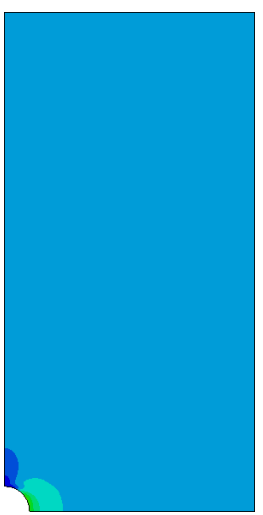

(d) $0.04 r_{h}, \bar{\sigma}=$ 0.36 .

Figure 8: Contours of the reduced microdamage ISV $S_{r}$, normalized by the maximum $S_{r}$ obtained from all simulations, immediately prior to failure initiation for four different mesh densities. 

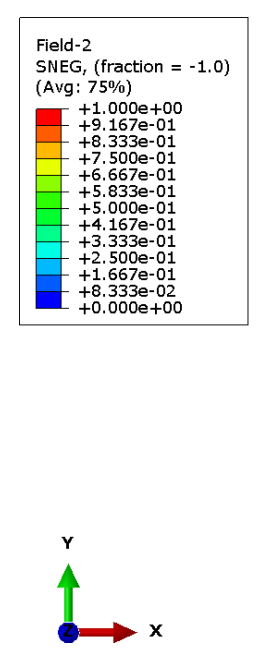

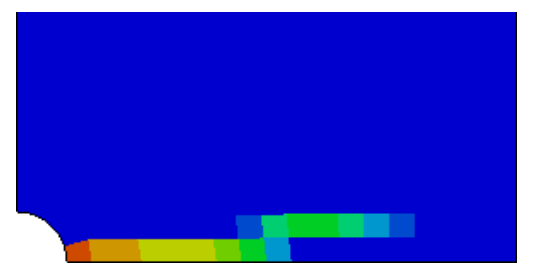

(a) $0.5 r_{h}, \bar{\sigma}=0.80$.

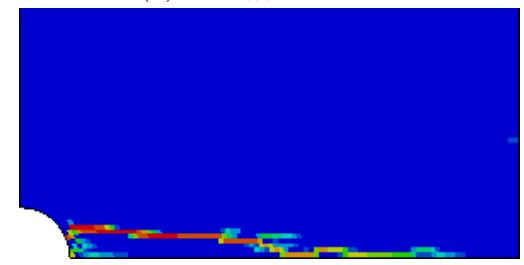

(c) $0.1 r_{h}, \bar{\sigma}=0.82$.

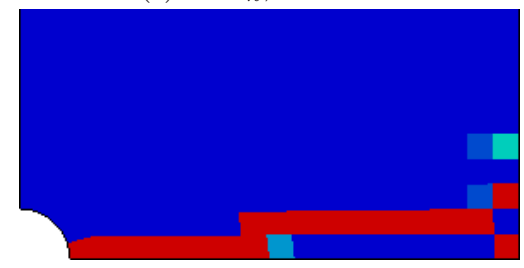

(e) $0.5 r_{h}, \bar{\sigma}=0.030$.

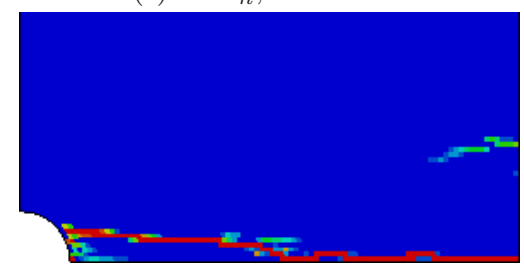

(g) $0.1 r_{h}, \bar{\sigma}=0.042$.

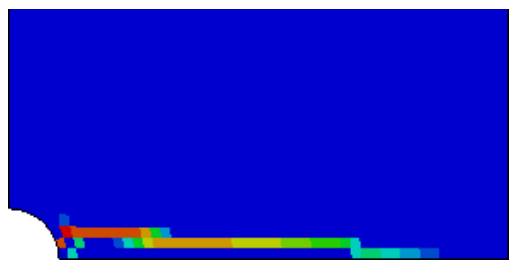

(b) $0.2 r_{h}, \bar{\sigma}=0.81$.

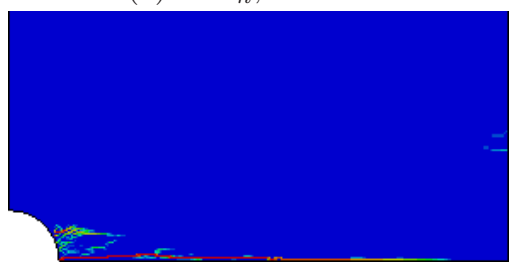

(d) $0.04 r_{h}, \bar{\sigma}=0.82$.

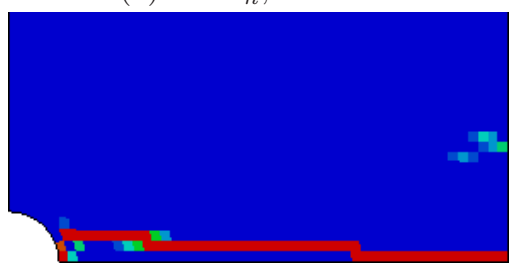

(f) $0.2 r_{h}, \bar{\sigma}=0.018$.

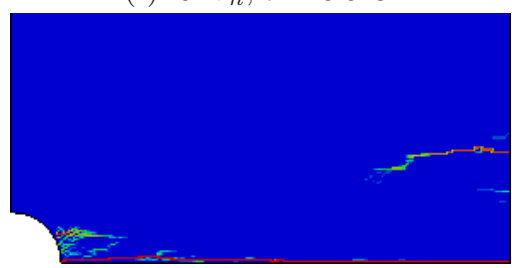

(h) $0.04 r_{h}, \bar{\sigma}=0.242$.

Figure 9: Contours of the transverse degradation parameter $D_{I}^{m}$, indicative of the transverse crack path in the specimens. The contours (a-d) are presented at the ultimate load achieved by the specimens and (e-h) after the specimens have lost their load carrying capability. 


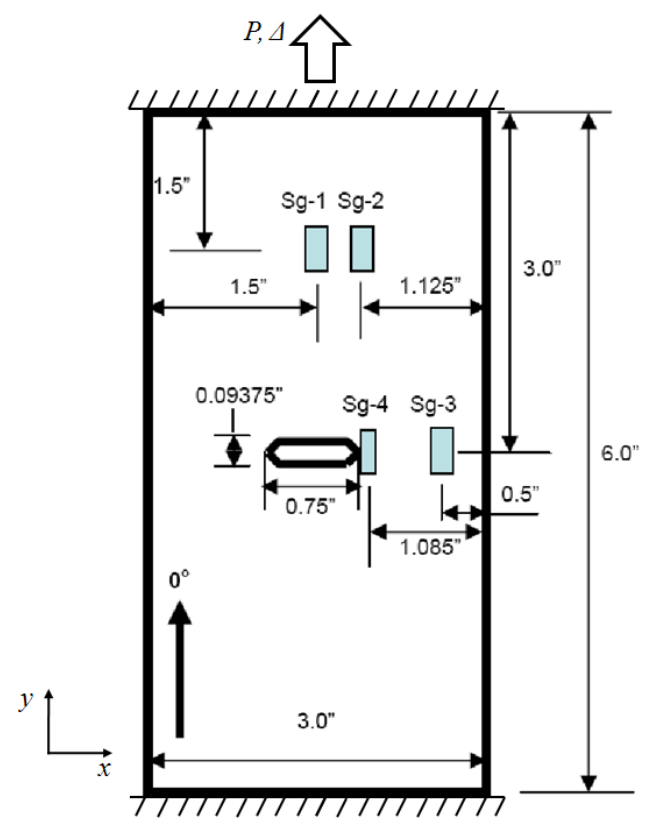

Figure 10: Geometry, boundary conditions, and strain gage (Sg) locations of CNPs tested at NASA LaRC. ${ }^{39}$

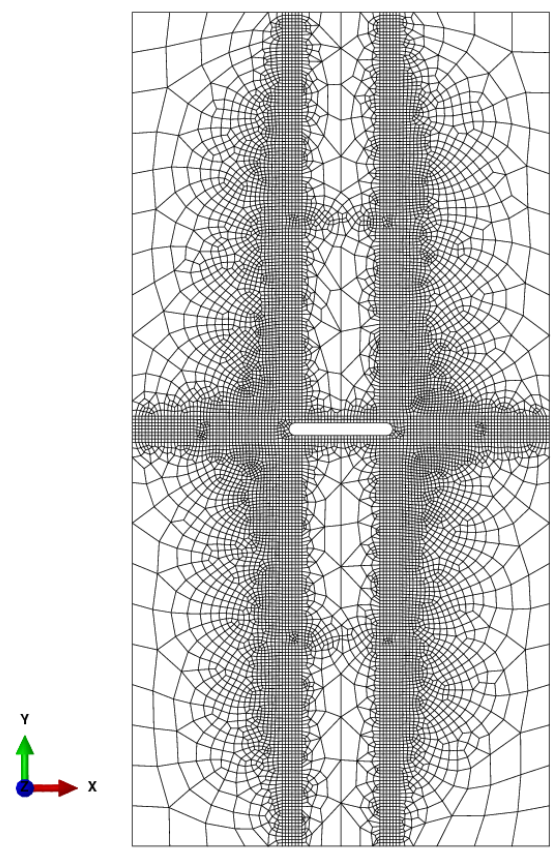

Figure 11: FEM mesh used to simulate tensile loading of CNPs. 


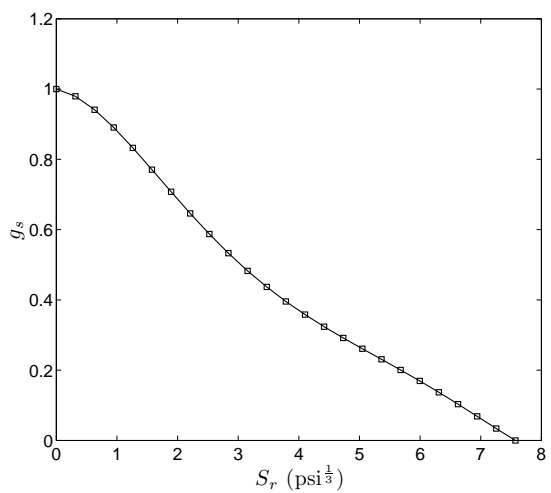

(a) Shear microdamage function obtained from $\left[ \pm 45^{\circ}\right]_{\mathrm{S}}$ angle-ply laminate.

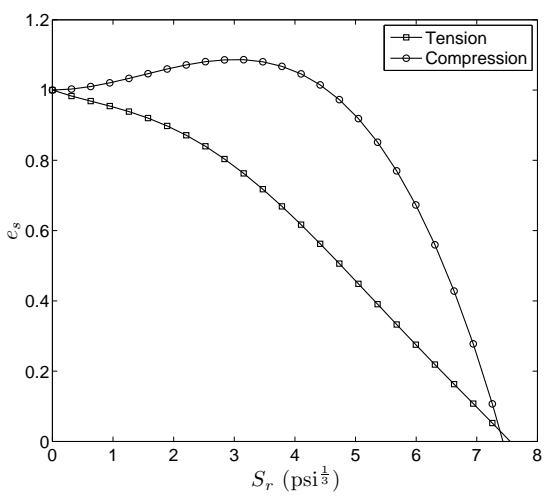

(b) Transverse tension and compression microdamage functions obtained by scaling data for AS4/3502 in Ref. 36.

Figure 12: Microdamage functions for T800/3900-2 used in FEM models.

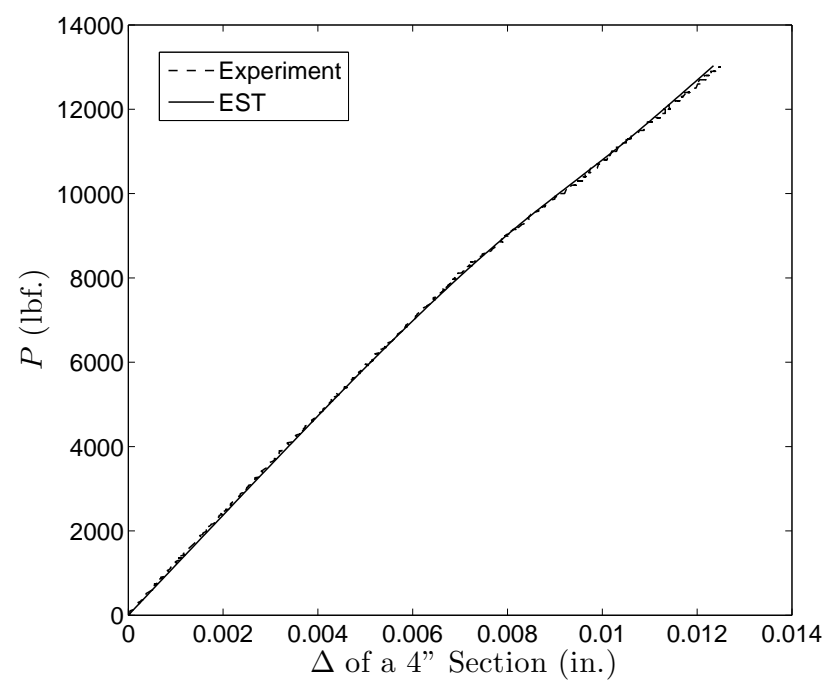

Figure 13: Applied load versus displacement of a 4" section for Laminate-1. 


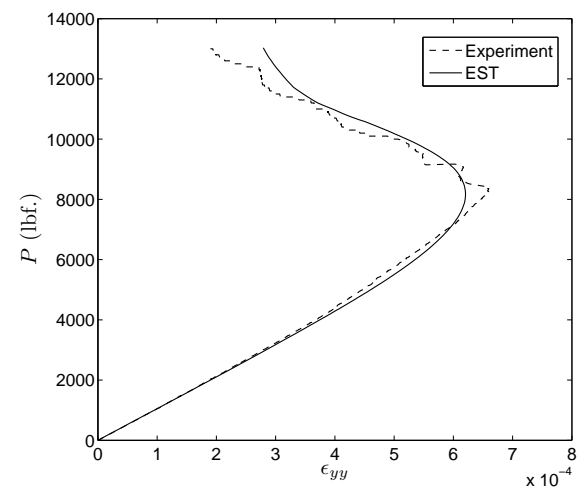

(a) $\mathrm{Sg}-1$.

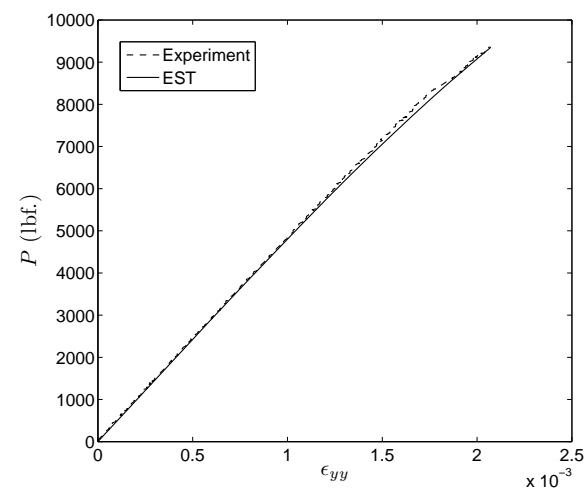

(c) $\mathrm{Sg}-3$.

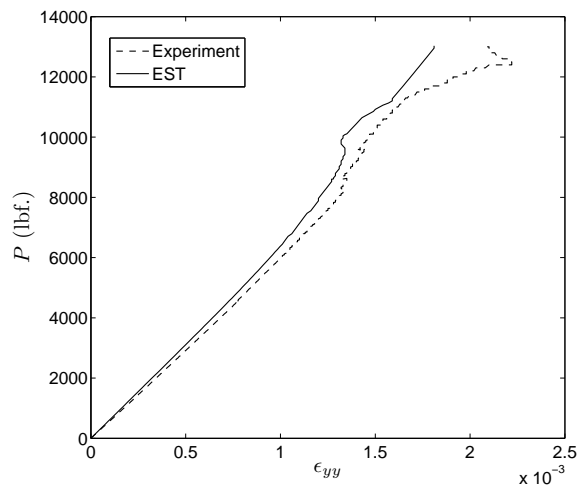

(b) $\mathrm{Sg}-2$.

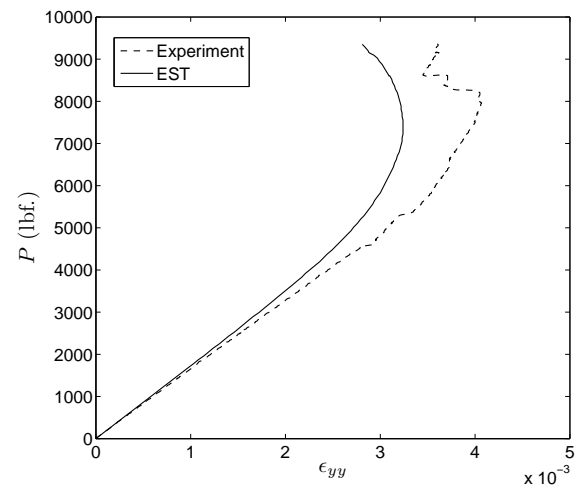

(d) $\mathrm{Sg}-4$.

Figure 14: Applied load versus local strain for Laminate-1.

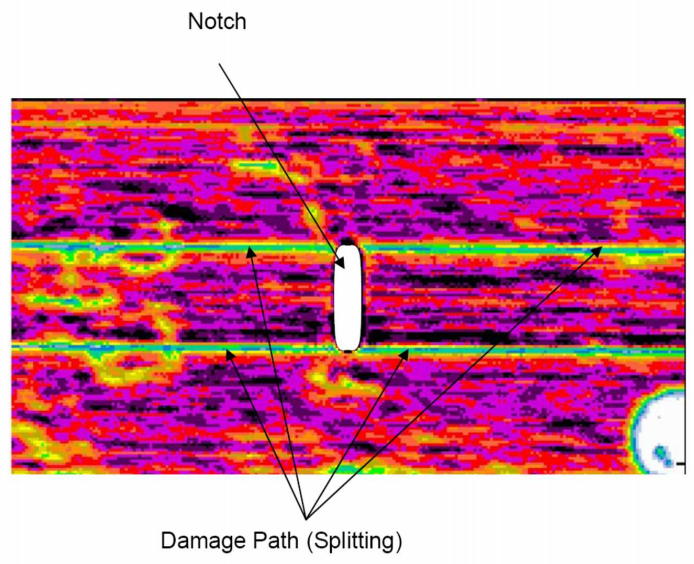

Figure 15: C-Scan of failed Laminate-1 specimen. ${ }^{39}$ 


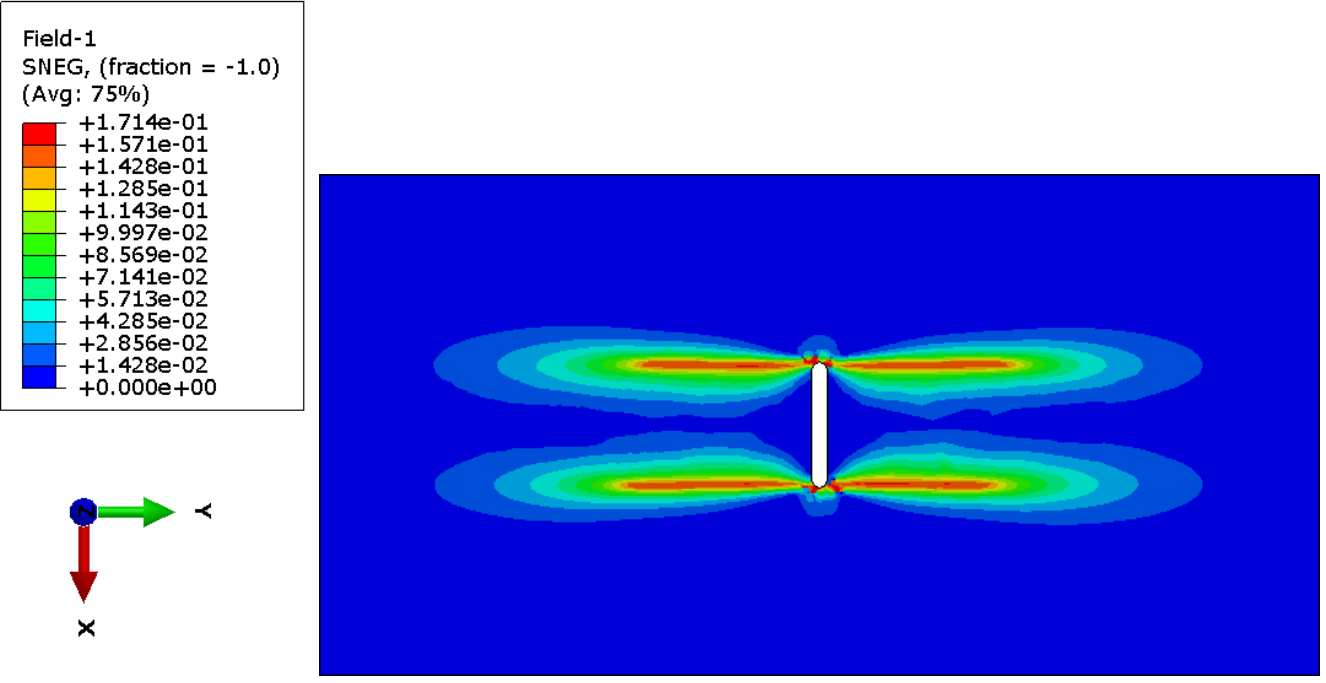

(a) $P=8,210 \mathrm{lbf}$.

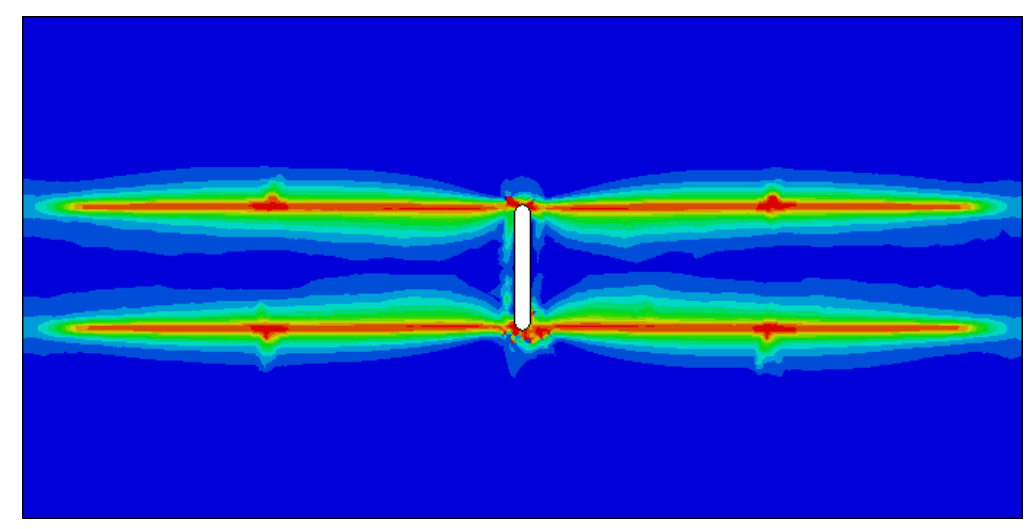

(b) $P=16,400 \mathrm{lbf}$.

Figure 16: Normalized matrix microdamage contour $\frac{S_{r}}{S_{r}^{\text {max }}}$ in Laminate-1. 


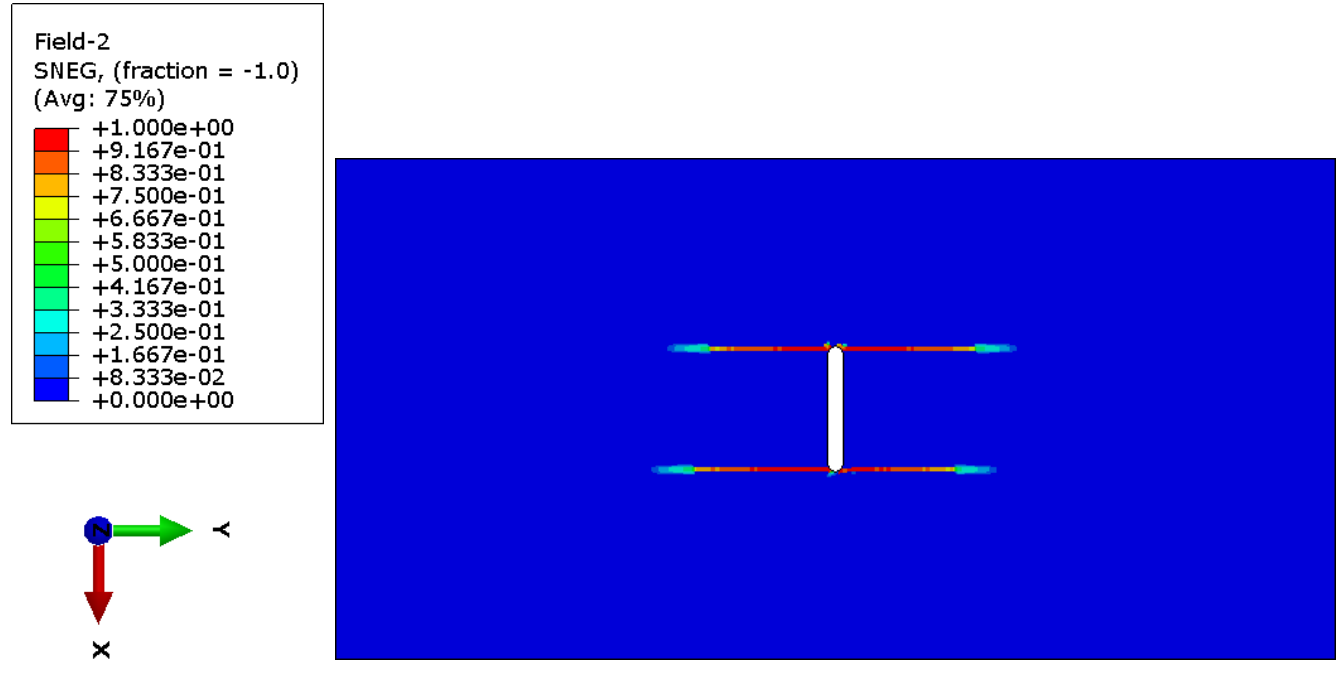

(a) $P=8,210 \mathrm{lbf}$.

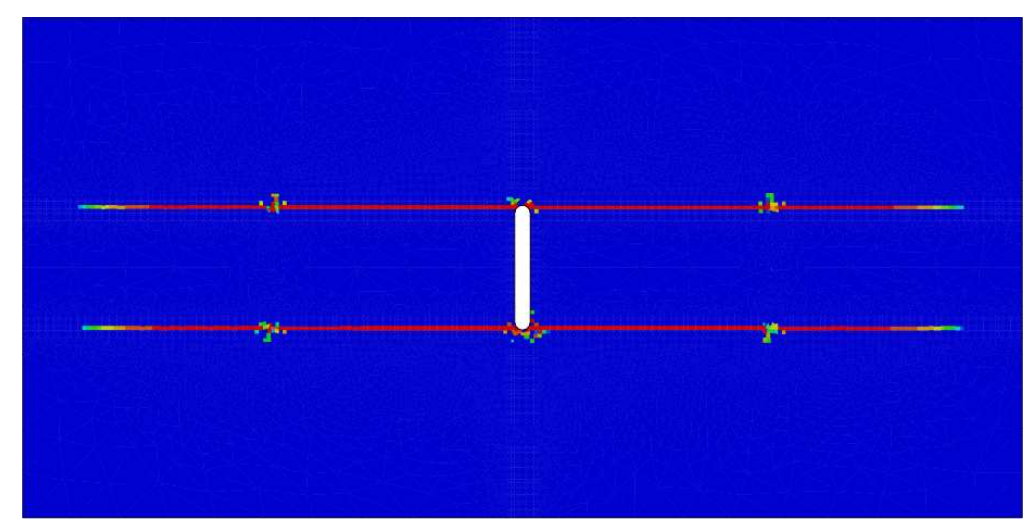

(b) $P=16,400 \mathrm{lbf}$.

Figure 17: Matrix shear failure degradation $D_{I I}^{m}$ in Laminate-1. 


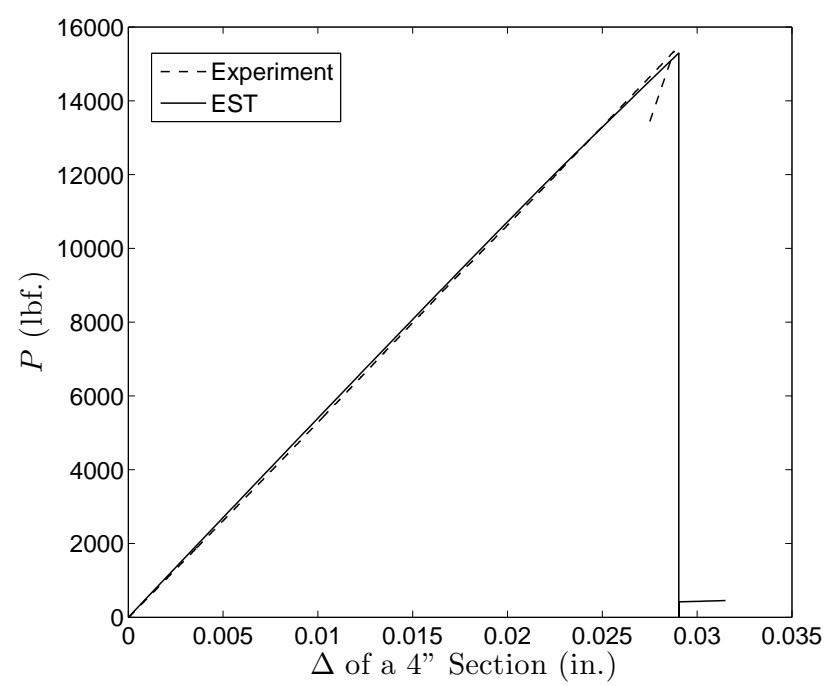

Figure 18: Applied load versus displacement of a 4" section for Laminate-2. 


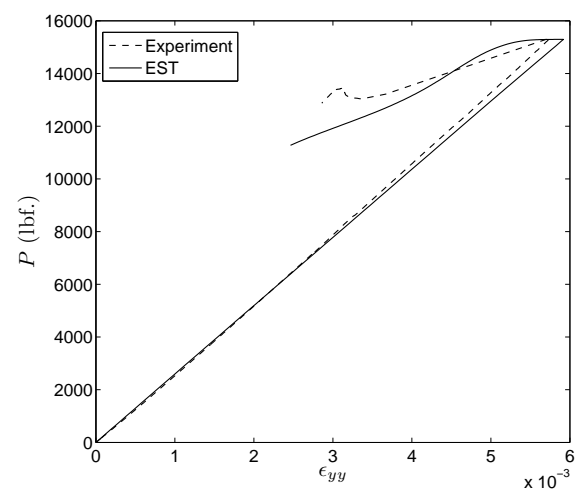

(a) $\mathrm{Sg}-1$.

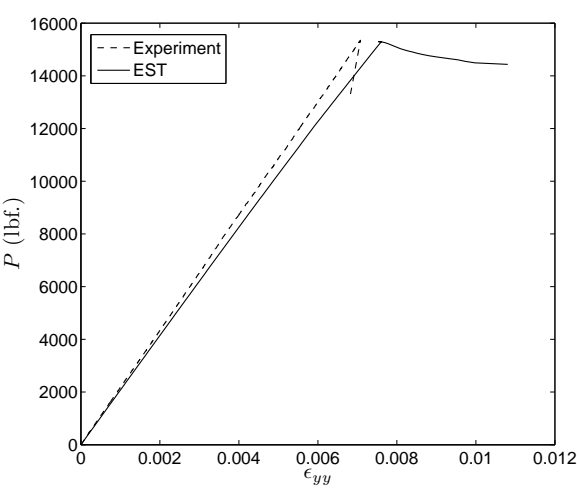

(c) $\mathrm{Sg}-3$.

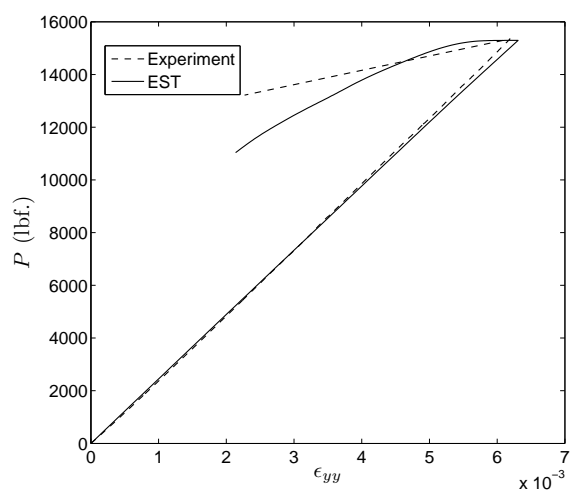

(b) Sg-2.

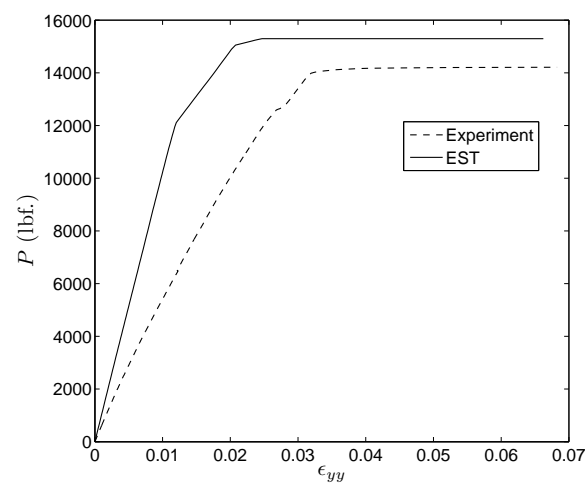

(d) $\mathrm{Sg}-4$.

Figure 19: Applied load versus local strain for Laminate-2.

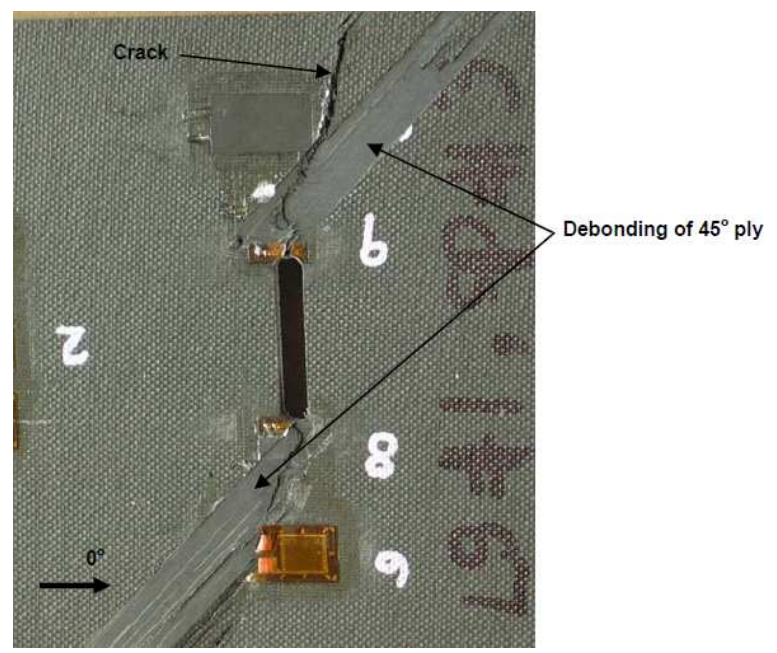

Figure 20: Photograph of failed Laminate-2 specimen. ${ }^{39}$ 

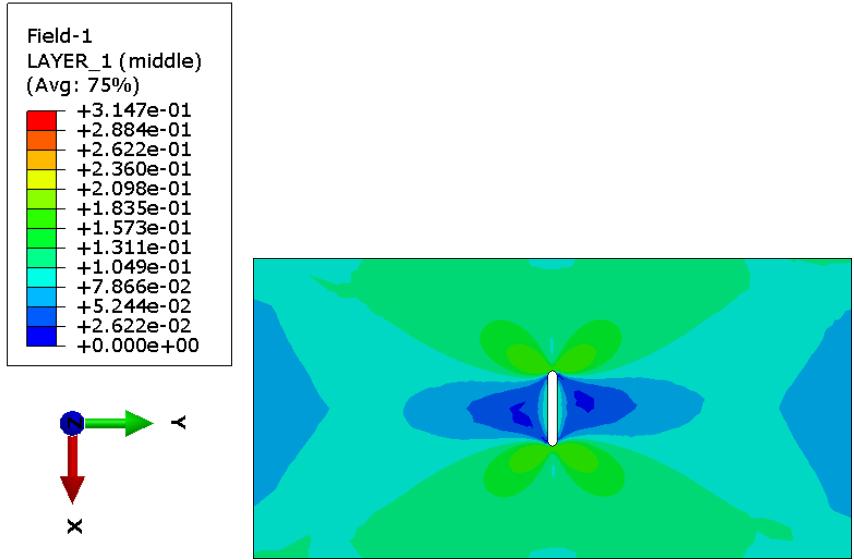

(a) $45^{\circ}$ Layer.

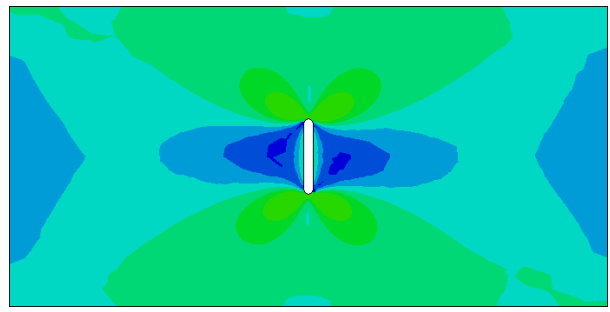

(c) $-45^{\circ}$ Layer.

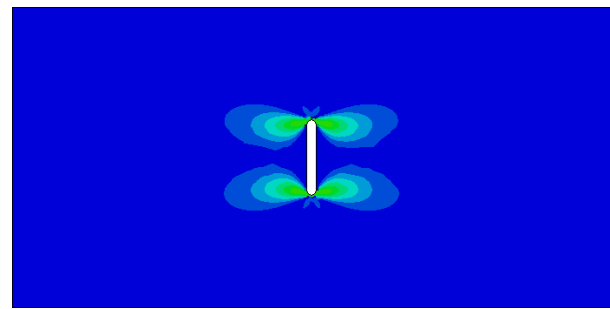

(b) $0^{\circ}$ Layer.

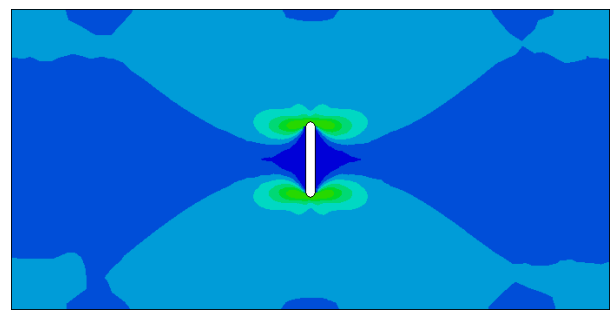

(d) $90^{\circ}$ Layer.

Figure 21: Normalized matrix microdamage contour $\frac{S_{r}}{S_{r}^{\text {max }}}$ in Laminate-2 just prior to first axial failure initiation $P=8,640 \mathrm{lbf}$. 

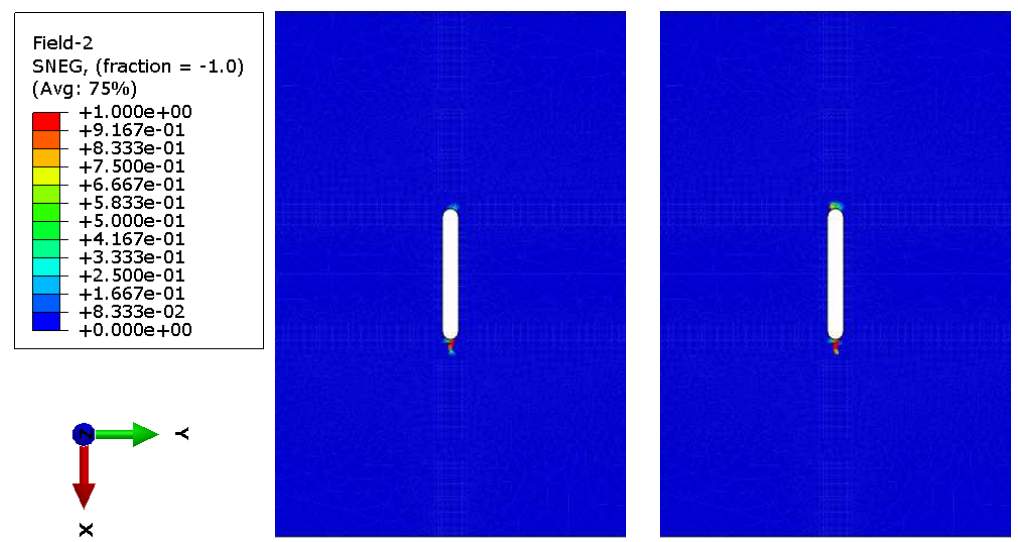

(a) $45^{\circ}$ Layer.

(b) $0^{\circ}$ Layer.
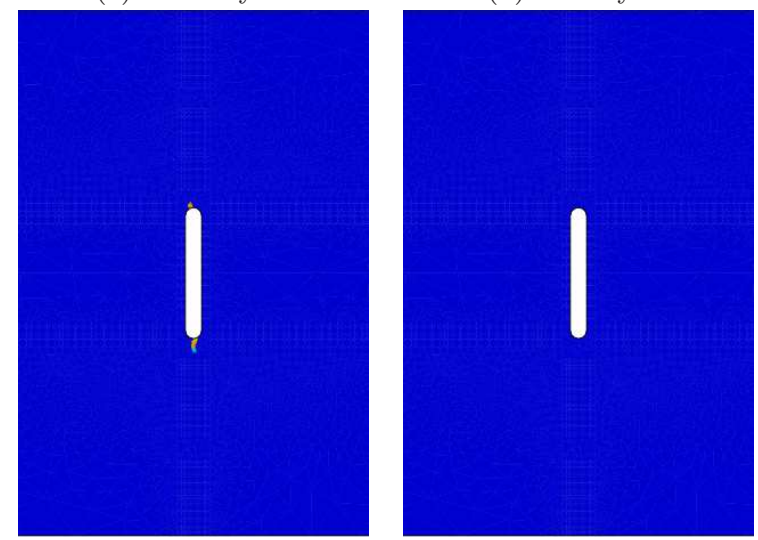

(c) $-45^{\circ}$ Layer.

(d) $90^{\circ}$ Layer.

Figure 22: Fiber failure degradation $D_{I}^{f}$ in Laminate-2 at ultimate load $P=15,300 \mathrm{lbf}$ (magnified view of region near notch). 


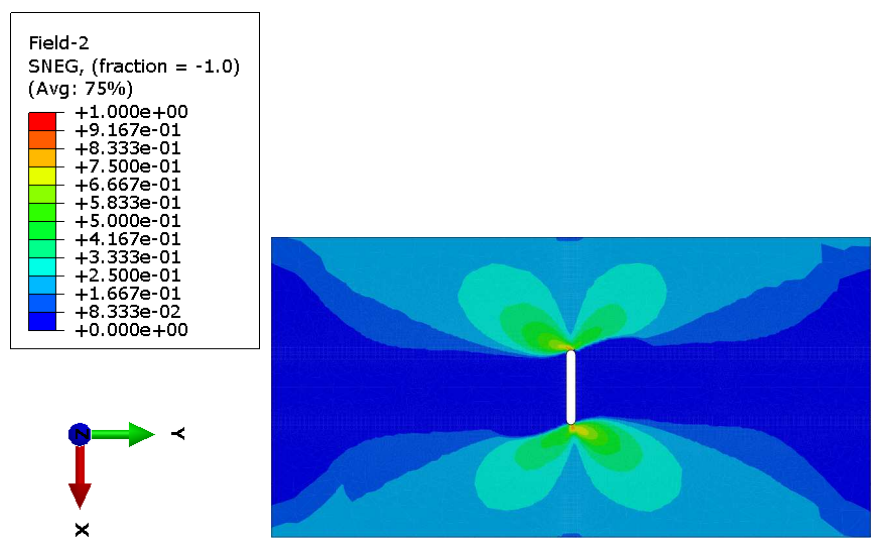

(a) $45^{\circ}$ Layer.

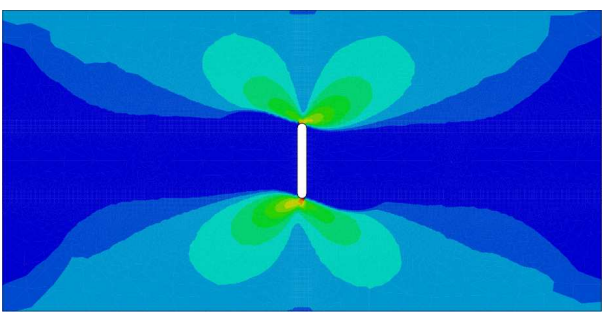

(c) $-45^{\circ}$ Layer.

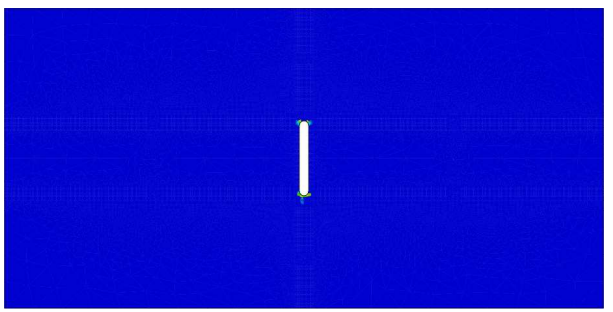

(b) $0^{\circ}$ Layer.

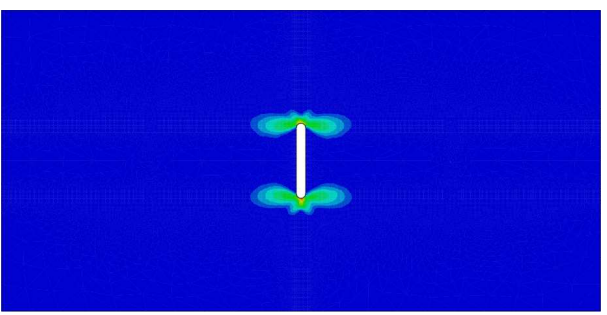

(d) $90^{\circ}$ Layer.

Figure 23: Transverse matrix failure degradation $D_{I}^{m}$ in Laminate-2 at ultimate load $P=15,300$ lbf. 

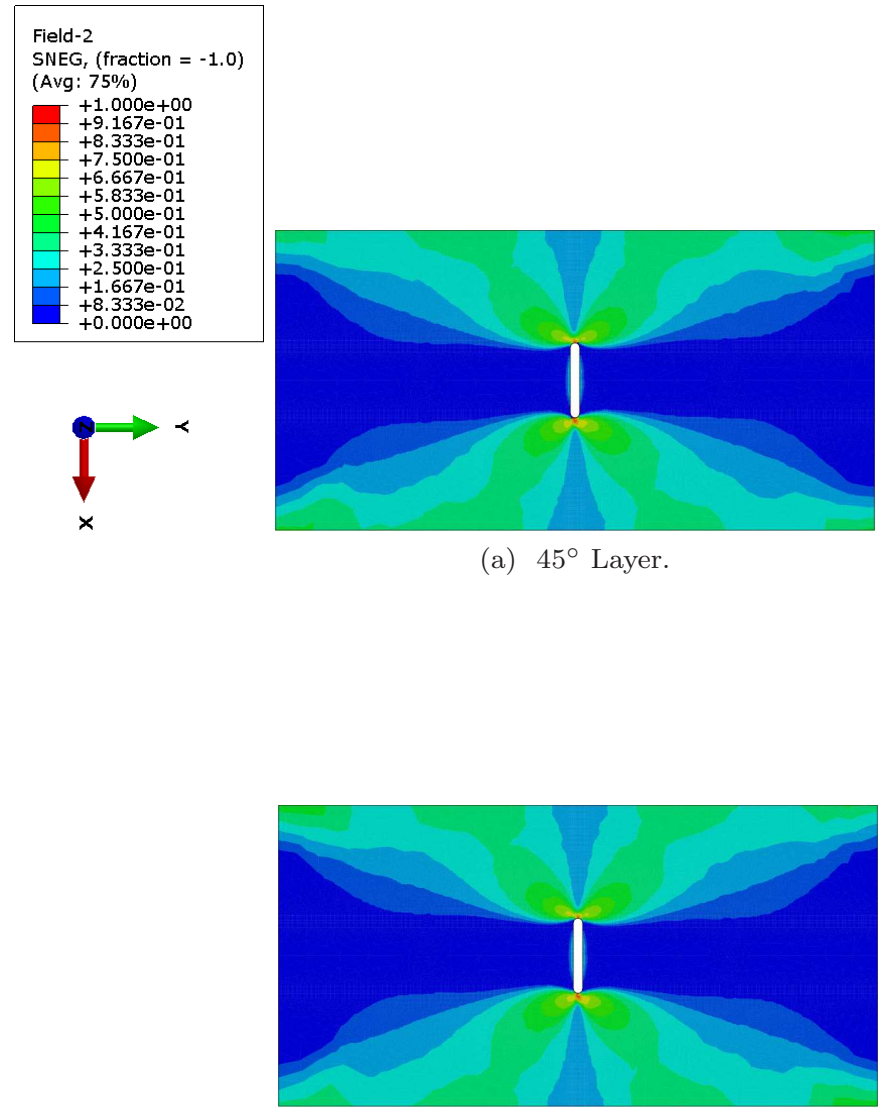

(c) $-45^{\circ}$ Layer.

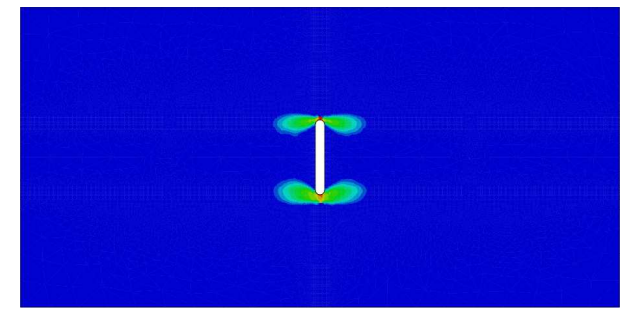

(b) $0^{\circ}$ Layer.

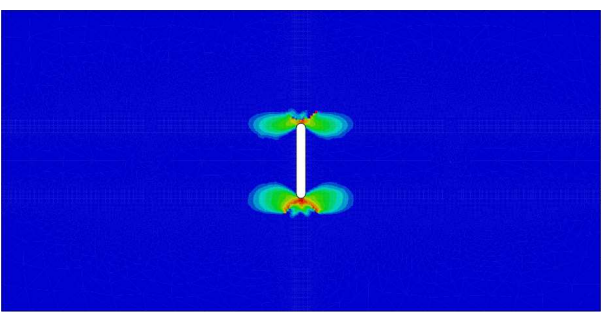

(d) $90^{\circ}$ Layer.

Figure 24: Shear matrix failure degradation $D_{I I}^{m}$ in Laminate-2 at ultimate load $P=15,300 \mathrm{lbf}$. 

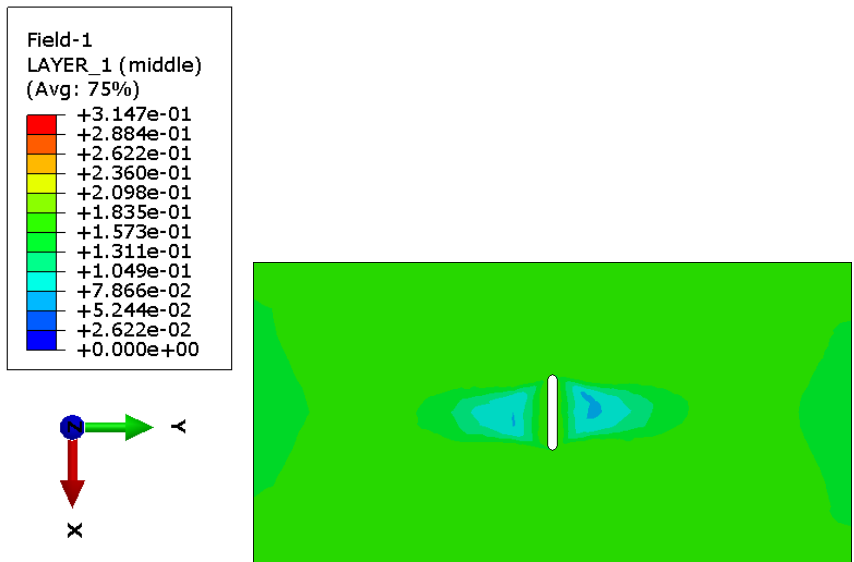

(a) $45^{\circ}$ Layer.

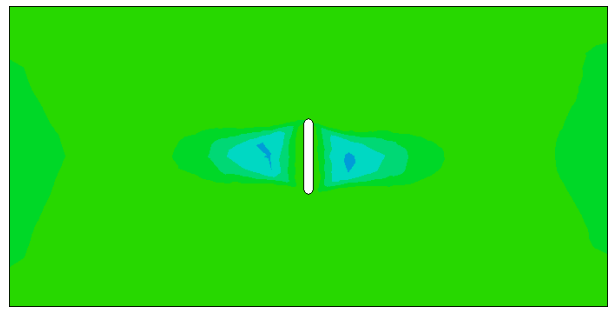

(c) $-45^{\circ}$ Layer.

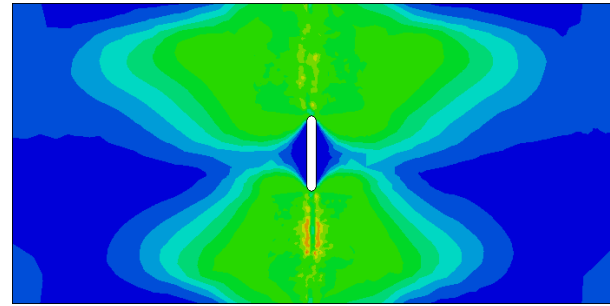

(b) $0^{\circ}$ Layer.

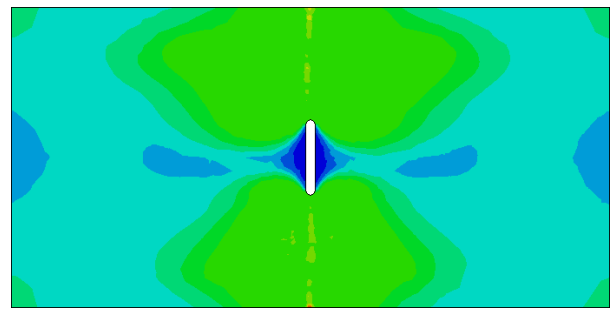

(d) $90^{\circ}$ Layer.

Figure 25: Normalized matrix microdamage contour $\frac{S_{r}}{S_{r}^{\text {max }}}$ in Laminate-2 after specimen has lost load carrying capability. 


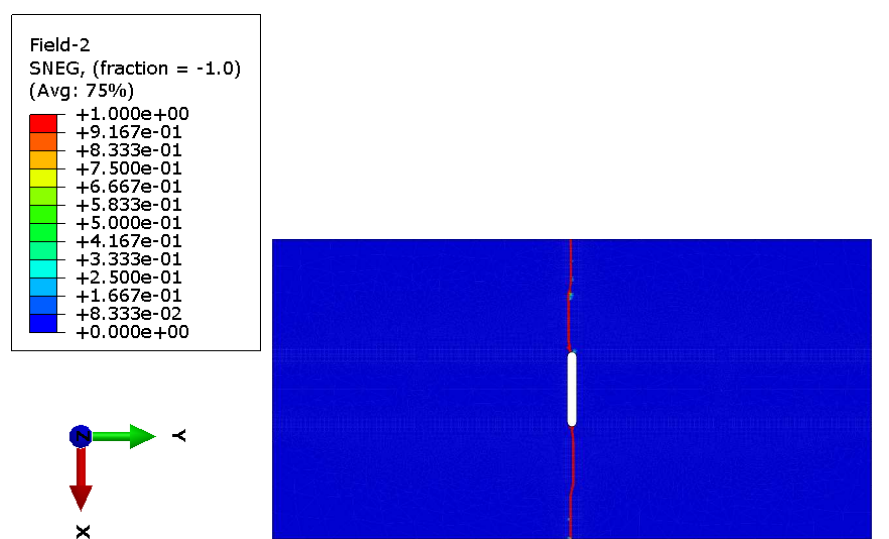

(a) $45^{\circ}$ Layer.

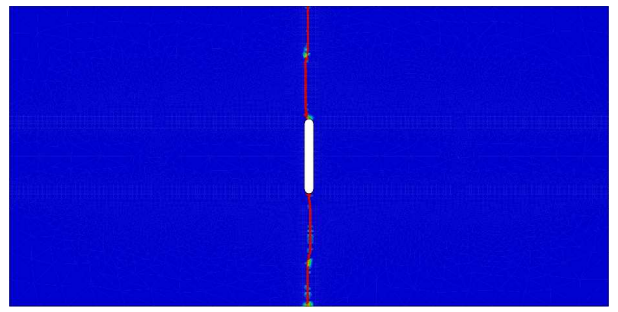

(b) $0^{\circ}$ Layer.

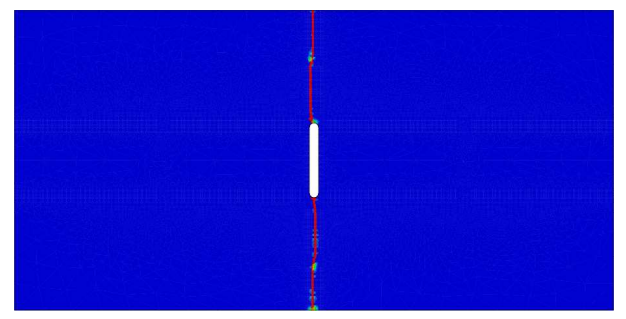

(c) $-45^{\circ}$ Layer.

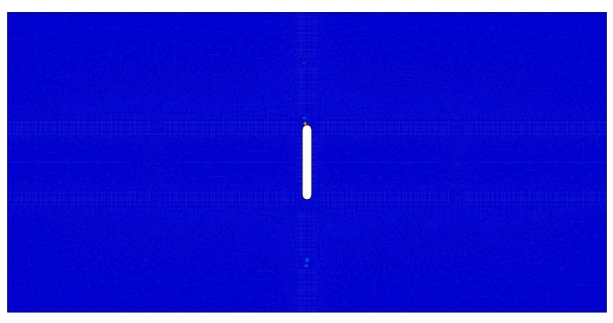

(d) $90^{\circ}$ Layer.

Figure 26: Fiber failure degradation $D_{I}^{f}$ in Laminate-2 after specimen has lost load carrying capability. 


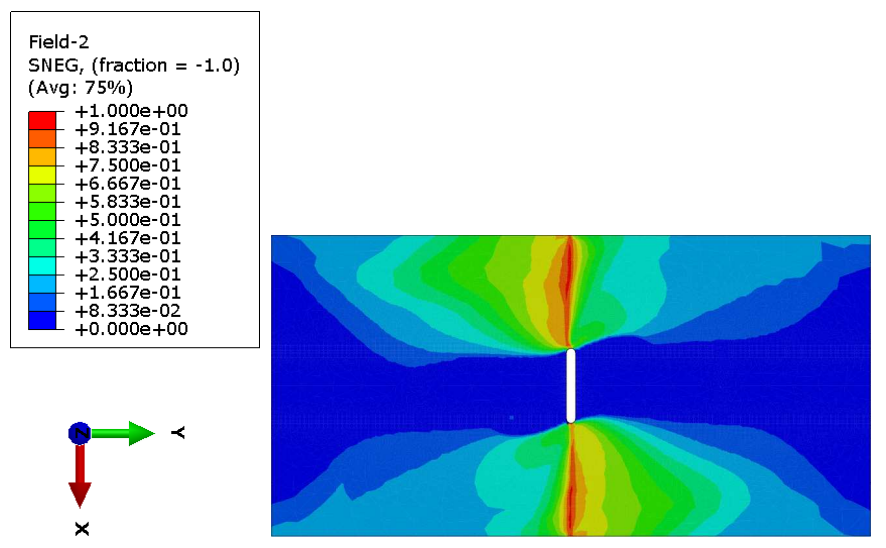

(a) $45^{\circ}$ Layer.

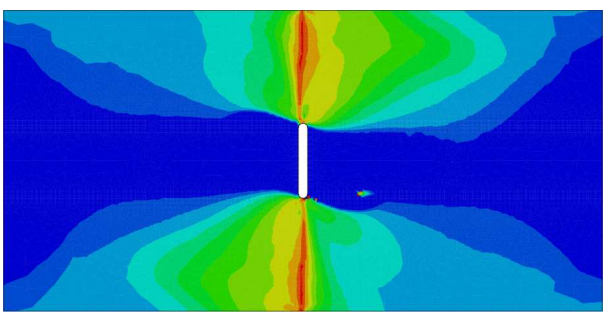

(c) $-45^{\circ}$ Layer.

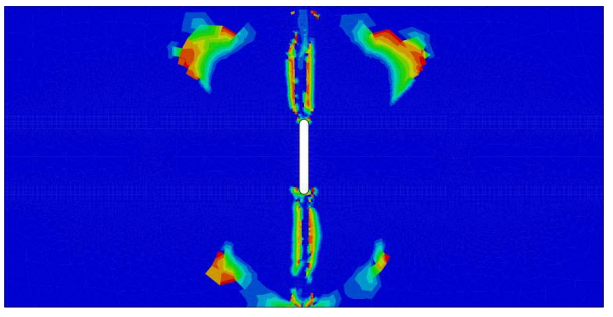

(b) $0^{\circ}$ Layer.

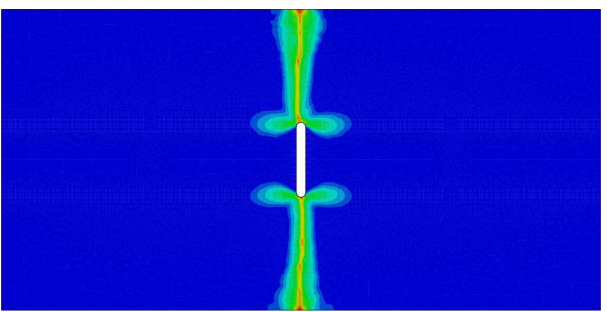

(d) $90^{\circ}$ Layer.

Figure 27: Transverse matrix failure degradation $D_{I}^{m}$ in Laminate-2 after specimen has lost load carrying capability. 


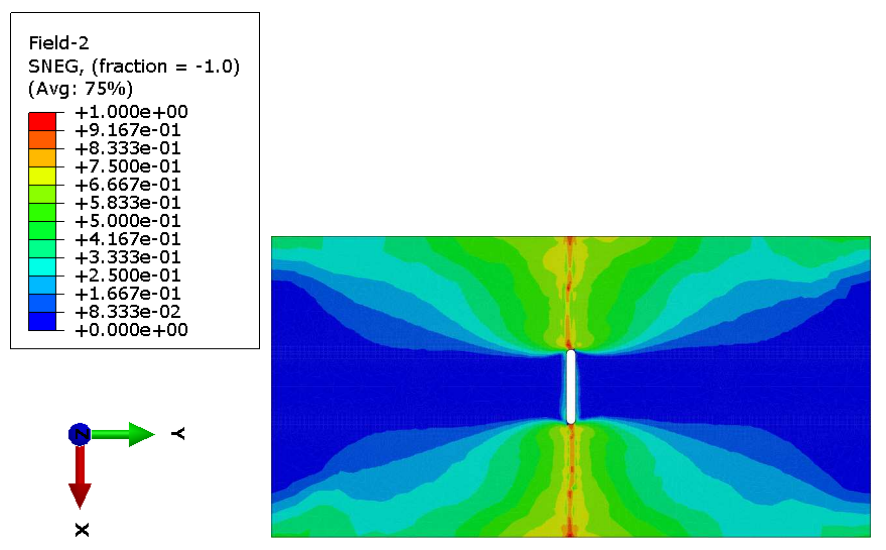

(a) $45^{\circ}$ Layer.

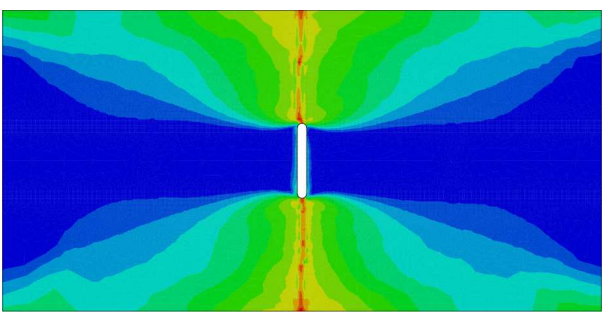

(c) $-45^{\circ}$ Layer.

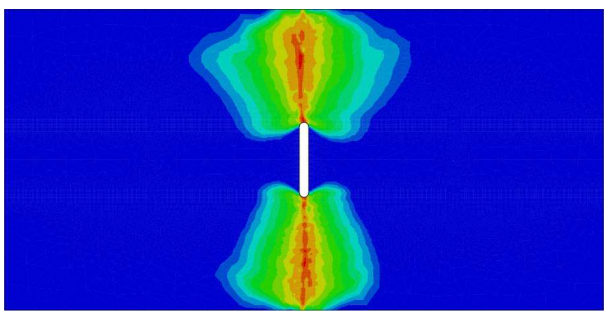

(b) $0^{\circ}$ Layer.

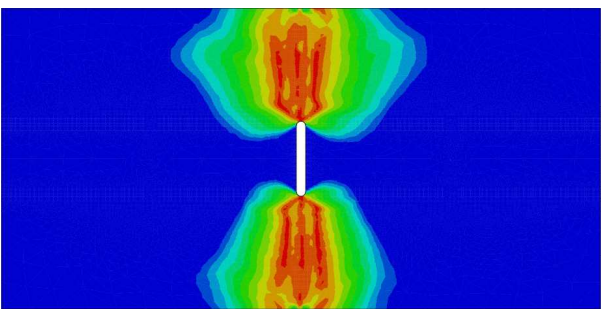

(d) $90^{\circ}$ Layer.

Figure 28: Shear matrix failure degradation $D_{I I}^{m}$ in Laminate-2 after specimen has lost load carrying capability. 


\section{References}

${ }^{1}$ Kachanov, L. M., "On the creep fracture time," Izvestiya Akademii Nauk SSSR, Otdeleniya Tekhnika Nauk, Vol. 8, No. 1, 1958, pp. 26-31.

${ }^{2}$ Kachanov, L. M., Introduction to Continuum Damage Mechanics, Martinus-Nijhoff, Dordrecht, The Netherlands, 1986.

${ }^{3}$ Talreja, R., "A continuum mechanics cahracterization of damage in composite materials," P. Roy. Soc. Lond. A Mat., Vol. 4, 1985, pp. 335-375.

${ }^{4}$ Lemaitre, J. and Chaboche, J.-L., Mechanics of Solid Materials, Cambridge University Press, 1994.

${ }^{5}$ Lemaitre, J., A Course on Damage Mechanics, 2nd Ed., Springer-Verlag, Berlin, Heidelberg, New York, 1996.

${ }^{6}$ Krajcinovic, D., Damage Mechanics, Elsevier, New York, 1996.

${ }^{7}$ Voyiadjis, G. and Kattan, P. I., Damage Mechanics, Taylor and Francis, Boca Raton, FL, 2005.

${ }^{8}$ Dvorak, G. J., Laws, N., and Hejazi, M., "Analysis of progressive matrix cracking in composite laminates I. Thermoelastic properties of a ply with cracks," J. Compos. Mater., Vol. 19, 1985, pp. 216-234.

${ }^{9}$ Talreja, R., "Transverse cracking and stiffness reduction in composite laminates," J. Composite Materials, Vol. 19, 1985, pp. $355-275$.

${ }^{10}$ Laws, N. and Dvorak, G. J., "Progressive transverse cracking in composite laminates," J. Compos. Mater., Vol. 22, 1988, pp. 900-916.

${ }^{11}$ Lee, J.-W., Allen, D. H., and Harris, C. E., "Internal state variable approach for predicting stiffness reductions in fibrous laminated composites with matrix cracks," J. Compos. Mater., Vol. 23, 1989, pp. 1273-1291.

${ }^{12}$ Nairn, J. A., "Strain energy release rate of composite microcracking: A variational approach," J. Compos. Mater., Vol. 23, 1989, pp. 1106-1129.

${ }^{13}$ Tan, S. C. and Nuismer, R. J., "A theory for progressive matrix cracking in composite laminates," J. Compos. Mater., Vol. 23, 1989, pp. 1029-1047.

${ }^{14}$ Gudmundson, P. and Östlund, S., "First order analysis of stiffness reduction due to matrix cracking," J. Compos. Mater., Vol. 26, 1992, pp. 1009-1030.

${ }^{15}$ McCartney, L. N., "Theory of stress transfer in a $0^{\circ}-90^{\circ}-0^{\circ}$ cross-ply laminate containing a parallel array of transverse cracks," J. Mech. Phys. Solids, Vol. 40, No. 1, 1992, pp. 27-68.

${ }^{16}$ McCartney, L. N., "Predicting transverse crack formation in cross-ply laminates," Compos. Sci. Technol., Vol. 58, 1998, pp. 1069-1081.

${ }^{17}$ Allen, D. H., Harris, C. E., and Groves, S. E., "A thermomechanical constitutive theory for elastic composites with distributed damage - I. Theoretical development," Int. J. Solids Struct., Vol. 23, No. 9, 1987, pp. 1301-1318.

${ }^{18}$ Allen, D. H., Harris, C. E., and Groves, S. E., "A thermomechanical constitutive theory for elastic composites with distributed damage - II. Aplication to matrix cracking in laminated composites," Int. J. Solids Struct., Vol. 23, No. 9, 1987, pp. 1319-1338.

${ }^{19}$ Talreja, R., editor, Vol. 9 of Composite Materials Series, Elsevier Science B.V., Amsterdam, The Netherlands, 1994.

${ }^{20}$ Paas, M. H. J. W., Schreurs, P. J. G., and Brekelmans, W. A. M., "A continuum approach to brittle and fatigue damage: Theory and numerical procedures," Int. J. Solids Struct., Vol. 30, No. 4, 1992, pp. 579-599.

${ }^{21}$ Matzenmiller, A., Lubliner, J., and Taylor, R. L., "A constitutive model for anisotropic damage in fiber-composites," Mech. Mater., Vol. 20, No. 2, 1995, pp. 125-152.

${ }^{22}$ Bednarcyk, B. A., Aboudi, J., and Arnold, S. M., "Micromechanics modeling of composites subjected to multiaxial progressive damage in the constituents," AIAA J., Vol. 48, 2010, pp. 1367-1378.

${ }^{23}$ Puck, A. and Schürmann, H., "Failure analysis of FRP laminates by means of physically based phenomenological models," Comps. Sci. Technol., Vol. 58, 1998, pp. 1045-1067.

${ }^{24}$ Puck, A. and Schürmann, H., "Failure analysis of FRP laminates by means of physically based phenomenological models," Comps. Sci. Technol., Vol. 62, 2002, pp. 1633-1622.

${ }^{25}$ Pinho, S. T., Dávila, C. G., Camanho, P. P., and Iannucci, L., "Failure models and criteria for FRP under in-plane or three-dimensional stress states including shear non-linearity," NASA/TM 2005-213530, 2005.

${ }^{26}$ Bažant, Z. and Cedolin, L., "Blunt crack band propagation in finite element analysis," J. Eng. Mech. Div.-ASCE, Vol. 105, 1979, pp. 297-315.

${ }^{27}$ Pietruszczak, S. and Mroz, Z., "Finite element analysis of deformation of strain-softening materials," Int. J. Numer. Methods Eng., Vol. 17, 1981, pp. 327-334.

${ }^{28}$ Bažant, Z. P., "Crack band model for fracture of geomaterials," Proceedings of the $4^{\text {th }}$ International Conference on Numerical Methods in Geomechanics, Edmonton, Canada, 1982.

${ }^{29}$ Bažant, Z. P. and Oh, B. H., "Crack band theory for fracture of concrete," Mater. and Struct., Vol. 16, 1983, pp. 155-77.

${ }^{30}$ de Borst, R. and Nauta, P., "Non-orthogonal cracks in a smeared finite element model," Eng. Comput., Vol. 2, 1985, pp. $35-46$.

${ }^{31}$ Spencer, B. W., Finite Elements with Embedded Discontinuities for Modeling Reinforced Concrete Members, Ph.D. thesis, Brigham Young University, Provo, UT, 2002.

${ }^{32}$ Lamborn, M. J. and Schapery, R. A., "An investigation of deformation path-independence of mechanical work in fiberreinforced plastics," Proceedings of the Fourth Japan-U.S. Conference on Composite Materials, Technomic Publishing Co., Inc., Lancaster, PA, 1988.

${ }^{33}$ Schapery, R. A., "Mechanical characterization and analysis of inelastic composite laminates with growing damage," Mechanics \& Materials Center Report 5762-89-10, Texas A \& M University, College Station, TX 77804, 1989.

${ }^{34}$ Schapery, R. A., "A theory of mechanical behaviour of elastic media with growing damage and other changes in structure," J. Mech. Phys. Solids, Vol. 38, No. 2, 1990, pp. 1725-1797. 
${ }^{35}$ Lamborn, M. J. and Schapery, R. A., "An investigation of the existence of a work potential for fiber-reinforced plastic," J. Compos. Mater., Vol. 27, 1993, pp. 352-382.

${ }^{36}$ Sicking, D. L., Mechanical Characterization of Nonlinear Laminated Composites with Transverse Crack Growth, Ph.D. thesis, Texas A\&M University, College Station, TX, 1992.

${ }^{37}$ Schapery, R. A. and Sicking, D. L., "A theory of mechanical behaviour of elastic media with growing damage and other changes in structure," Mechanical Behaviour of Materials, edited by A. Bakker, Delft University Press, Delft, The Netherlands, 1995, pp. 45-76.

${ }^{38}$ Pineda, E. J., Waas, A. M., Bednarcyk, B. A., and Collier, C. S., "Computational implementation of a thermodynamically based work potential model for progressive microdamage and transverse cracking in fiber-reinforced laminates," 51st AIAA/ASME/ASCE/AHS/ASC Structures, Structural Dynamics, and Materials Conference, Orlando, FL, 12-15 April 2010.

${ }^{39}$ Bogert, P. B., Satyanarayana, A., and Chunchu, P. B., "Comparison of damage path predicions for composite laminates by explicit and standard finite element analysis tool," $47^{\text {th }}$ AIAA Structures, Structural Dynamics, and Materials Conference, 1-4 May 2006.

${ }^{40}$ Satyanarayana, A., Bogert, P. B., and Chunchu, P. B., "The effect of delamination on damage path and failure load prediction for notched composite laminates," $48^{\text {th }}$ AIAA Structures, Structural Dynamics, and Materials Conference, $23-26$ April 2007.

${ }^{41}$ Schapery, R. A., "Prediction of compressive strength and kink bands in composites using a work potential," Int. J. Solids Structures, Vol. 32, No. 6, 1995, pp. 739-765.

${ }^{42}$ Basu, S., Waas, A. M., and Ambur, D. R., "Compressive failure of fiber composites under multiaxial loading," Int. J. Solids Structures, Vol. 44, No. 9, 2006, pp. 2648-2676.

${ }^{43}$ Pineda, E. J., Waas, A. M., Bednarcyk, B. A., Collier, C. S., and Yarrington, P. W., "Progressive damage and faiure modeling in notched laminated fiber reinforced composites," Int. J. Fract., Vol. 158, 2009, pp. 125-143.

${ }^{44}$ Hinterhoelzl, A. and Schapery, R. A., "FEM implementation of a three-dimensional visoelastic constitutive model for particulate composites with damage growth," Mech. Time-Depend. Mat., Vol. 8, No. 1, 2004, pp. 65-94.

${ }^{45}$ Rice, J. R., "Inelastic constitutive relations for solids: an internal-variable theory and its application to metal plasticity," J. Mech. Phys. Solids, Vol. 19, 1971, pp. 433-455.

${ }^{46} \mathrm{Ng}$, W. H., Salvi, A. G., and Waas, A. M., "Characterization of the in-situ non-linear shear response of laminated fiber-reinforced composites," Compos. Sci. Technol., Vol. 70, No. 7, 2010, pp. 1126-1134.

${ }^{47}$ Yang, Q. and Cox, B., "Cohesive models for damage evolution in laminated composites," Int. J. Frac., Vol. 133, 2005, pp. 107-137.

${ }^{48}$ Noda, J., Okabe, T., Takeda, N., and Shimizu, M., "Tensile strength of CFRP cross-ply laminates containing transverse cracks," Adv. Comps. Mater., Vol. 15, No. 1, 2006, pp. 81-93.

${ }^{49}$ Green, B. G., Wisnom, M. R., and Hallett, S. R., "An experimental investigation into the tensile strength scaling of notched composites," Compos.: Part A, Vol. 38, 2007, pp. 867-878.

${ }^{50}$ McCartney, L. N., "Mechanics for the growth of bridged cracks in composite materials: Part I. Basic Principles," J. Compos. Technol. \& Res., Vol. 14, No. 3, 1992, pp. 133-154.

${ }^{51}$ Hallett, S. R., Jiang, W.-G., Khan, B., and Winsom, M. R., "Modelling the interaction between matrix cracks and delamination damage in scaled quasi-isotropic specimens," Compos. Sci. and Technol., Vol. 68, 2008, pp. 80-89.

${ }^{52}$ Basu, S., Computational Modeling of Progrssive Failure and Damage in Composite Laminates, Ph.D. thesis, University of Michigan, Ann Arbor, MI, 2005.

${ }^{53}$ Hashin, Z. and Rotem, A., "A fatigue failure criterion for fiber reinforced composite materials," J. Composite Materials, Vol. 7, 1973, pp. 448-464.

${ }^{54}$ Gustafson, P. A. and Waas, A. M., "The influence of adhesive constitutive parameters in cohesive zone finite element models of adhesively bonded joints," Int. J. Solids Struct., 2009, pp. 2201-2215.

${ }^{55}$ Xie, D. E., Salvi, A., Sun, C. E., Waas, A. M., and Caliskan, A., "Discrete cohesive zone model to simulate static fracture in 2-D triaxially braided carbon fiber composites," J. Compos. Mater., Vol. 40, No. 22, 2006.

${ }^{56}$ Gustafson, P. A., Analytical and Experimental Methods for Adhesively Bonded Joints Subjected to High Temperatures, Ph.D. thesis, University of Michigan, Ann Arbor, MI, 2008.

${ }^{57}$ Turon, A., Camanho, P. P., Costa, J., and Dávila, C. G., "A damage model for the simulation of delamination in advanced composites under variable-mode loading," Mech. Mater., Vol. 38, No. 11, 2006.

${ }^{58}$ Hoek, E. and Bieniawski, Z. T., "Brittle rock fracture propagation in rock under compression," Int. J. Fract., Vol. 1, No. 3, 1965, pp. 137-155.

${ }^{59}$ Abaqus, Abaqus User's Manual, Vol. 1-3, Version 6.10-1, Dassault Systèmes Simulia Corp., Providence, RI, 2008.

${ }^{60}$ Belytschko, T., Liu, W. K., and Moran, B., Nonlinear finite elements for continua and structures, Wiley, New York, 2000.

${ }^{61}$ Belytschko, T. and Mish, K., "Computability in non-linear solid mechanics," Int. J. Numer. Methods, Vol. 52, 2001, pp. $3-21$.

${ }^{62}$ Hughes, T. J. R., The Finite Element Method: Linear Static and Dynamic Finite Element Analysis, Dover Publications, Inc., 2000 . 